Article

\title{
Phenolic Profiles of Leaves, Grapes and Wine of Grapevine Variety Vranac (Vitis vinifera L.) from Montenegro
}

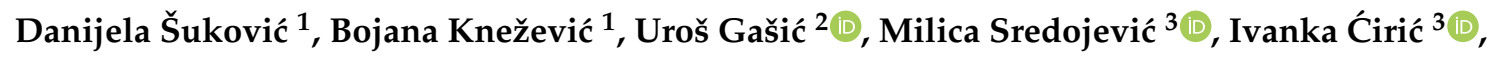 \\ Slavica Todić ${ }^{4}$, Jelena Mutić ${ }^{5}$ and Živoslav Tešić ${ }^{5, *}$ \\ 1 The Centre for Ecotoxicological Research, Bulevar Sarla de Gola 2, 81000 Podgorica, Montenegro; \\ danijela.sukovic@ceti.co.me (D.Š.); bojana.knezevic@ceti.co.me (B.K.) \\ 2 Institute for Biological Research "Siniša Stanković"-National Institute of Republic of Serbia, University of \\ Belgrade, Bulevar despota Stefana 142, 11060 Belgrade, Serbia; uros.gasic@ibiss.bg.ac.rs \\ 3 Innovation Center of the Faculty of Chemistry, University of Belgrade, P.O. Box 51, 11158 Belgrade, Serbia; \\ pantelicm@chem.bg.ac.rs (M.S.); ivankai@chem.bg.ac.rs (I.Ć.) \\ 4 Faculty of Agriculture, University of Belgrade, Nemanjina 6, 11080 Belgrade, Serbia; slavicat@agrif.bg.ac.rs \\ 5 Faculty of Chemistry, University of Belgrade, P.O. Box 51, 11158 Belgrade, Serbia; jmutic@chem.bg.ac.rs \\ * Correspondence: ztesic@chem.bg.ac.rs; Tel.: +381-113-336-733 or +381-112-639-357; Fax: +381-112-639-357
}

Received: 6 December 2019; Accepted: 22 January 2020; Published: 28 January 2020

\begin{abstract}
Vranac, an old autochthonous red grapevine variety of Montenegro, was first mentioned in a historical document published in the 15th century. As currently the study of indigenous varieties is of particular importance, the subject of this work was detailed characterization of phenolic compounds in the autochthonous grapevine variety Vranac, from the Montenegrin Podgorica subregion. Phenolic profiles of leaves, berries (skin, seeds, and pulp were examined separately) and young monovarietal wine were determined using ultra-high performance liquid chromatography (UHPLC) with linear trap quadrupole (LTQ) — Orbitrap XL mass spectrometry (MS). Total phenolic content (TPC) and radical scavenging activity (RSA) were higher for the grape seeds extracts, followed by extracts of grape skins and pulps. As expected, the total anthocyanin content (TAC) was higher in grape skin than in wine. A total of one hundred twenty nine compounds (forty two phenolic acids and their derivatives, twenty three flavan-3-ols, twenty one flavanols, five stilbenes and thirty eight anthocyanins) were identified in the investigated extracts. To our best knowledge, this is the first report of tentative identification of (epi)catechin 3-O-coumarate in grape seed and chalcan-flavan 3-ol dimers in wine and grape seed.
\end{abstract}

Keywords: vranac; grape; wine; LC/MS; polyphenolics; anthocyanins

\section{Introduction}

Content of phenolic compounds is one of the main factors in the quality of grapes and wine. Quantity and structure of phenolic compounds in grapes significantly affect the oenological potential of grapes and sensory attributes of the wine, influencing the color, astringency, bitterness, stability and age-ability of wines [1].

Different factors affect the polyphenolic profile, such as variety [2,3], climatic conditions and seasonal weather [4,5], vine health status [6], as well as vineyard management [7]. In addition, the polyphenols profile of wine is influenced by the winemaking techniques employed [5].

Bearing in mind the importance of the phenolic grape profile on the quality and sensory properties of wines and numerous factors affecting the phenolic profile, great interest in these compounds is quite justified [8]. Phenolic biological properties such as antioxidant, anti-inflammatory, cardioprotective, 
and cancer protective effects in human medicine have been studied [9]. The chemical nature and behavior of polyphenols contribute to the fingerprint of authenticity and typicality of the variety and area of origin [10].

The anthocyanin fingerprints of varietal wine have been suggested as an analytical tool for authenticity certification [11]. On the other hand, because the anthocyanin profile of the wine may be different from the anthocyanin profile of the grape and can be influenced by the wine production techniques applied, some authors challenge the use anthocyanin pattern of the grape skin and corresponding wine for this purpose [12]. Dimitrovska et al. [11] state that anthocyanin profile of the grape berry skin and produced wine is similar and the correlation between the grape and wine anthocyanin patterns could be confirmed. Some other factors such as the age of the wine may compromise the use anthocyanin fingerprinting in assessment of authenticity of wine.

The indigenous Balkan wine grape variety Vranac is widely spread in Montenegro, Serbia and mostly in North Macedonia where it represents a very important variety from an economic point of view [3].

The aim of this study was detailed characterization of the phenolic profile of the autochthonous grapevine variety Vranac, originating from the Podgorica subregion (Montenegro) using ultra-high performance liquid chromatography (UHPLC) in combination with high-resolution and multi stage mass spectrometry (MS). The composition of polyphenols was determined in leaves, different parts of berry (seed, skin, pulp), as well as in the young monovarietal wine with a view to promoting the varietal character of Vranac young wine and to advising on the most appropriate winemaking techniques. Further, detailed analysis of the phenolic profile of Vranac variety, with the already existing ampelographic and molecular characterization will contribute to a more complete characterization of this autochthonous variety.

\section{Materials and Methods}

\subsection{Chemicals}

Acetonitrile, methanol and formic acid (all MS grade), standards of phenolic compounds, and 6-hydroxy-2,5,7,8-tetramethylchroman-2-carboxylic acid (Trolox) were obtained from Sigma-Aldrich (Steinheim, Germany), while 2, 2-diphenyl-1-picrylhydrazyl (DPPH) was purchased from Fluka AG (Buch, Switzerland). Folin-Ciocalteu reagent and sodium carbonate were obtained from Merck (Darmstadt, Germany). Standard solutions and dilutions were prepared using ultrapure water (TKA Germany MicroPure water purification system, $0.055 \mu \mathrm{S} / \mathrm{cm}$ ). Syringe filters $(25 \mathrm{~mm}$, nylon membrane $0.45 \mu \mathrm{m}$ ) were purchased from Supelco (Bellefonte, PA, USA).

\subsection{Samples}

The Vranac berry samples were collected at technological maturity (September 2014) from three vineyards located at the "Ćemovsko" field location which belongs to the Montenegrin viticultural region. Leaf samples were collected from the same places as the grape samples from which the tested wine was made. The Geographic coordinate system (GPS) coordinates of the experimental site is $42.26^{\circ} \mathrm{N}, 19.16^{\circ} \mathrm{E}$ and elevation $40-70 \mathrm{~m}$ a.s.l. A sketch of all three vineyards is shown on Figure S1. The proximity of the sea to a large extent determines the mesoclimate of the "Ćemovsko" field. Sampling at "Aerodrom" location was performed in block IV at T2, T3 and T4. Sampling at the location "Šipčanik" was realized on T10, T11 and T12. Sampling at "Bunar" location was realized in Block II, T1. For the sample abbreviations see Table 1. This region is located on the southernmost part of the Adriatic coast. Sampling was performed by picking grape berries randomly distributed throughout different vines. Each sample consisted of three replicates, each of 100 randomly selected berries on both sides of canopy and from different parts of clusters. Before the analysis, frozen grape berries were separated into three tissues (skin, pulp, and seeds). The seeds, as well as leaf samples were washed with water and dried (in the dark, at $22^{\circ} \mathrm{C}$ ) for twenty days. Dry samples were ground into a powder. 


\subsection{Preparation of Extracts and Fractionation of the Skin Extract and Wine}

Procedures for extraction of phenolic compounds from grapes and leaves were described in literature [13,14]. Dry leaf and seed samples were extracted with $\mathrm{MeOH} / \mathrm{H}_{2} \mathrm{O}(70: 30, v / v)$ solution containing $0.1 \% \mathrm{HCl}$, while frozen grape skins and pulps were extracted with acidified $\mathrm{MeOH}(0.1 \%$ $\mathrm{HCl} v / v$ ). Extractions (in an ultrasonic bath during $60 \mathrm{~min}$, at room temperature, in shade) were repeated three times. All three fractions were merged into one total extract and evaporated (at $40^{\circ} \mathrm{C}$ ) on a vacuum evaporator IKA RV 8 (IKA ${ }^{\circledR}$-Werke $\mathrm{GmbH}$ and Co. KG, Staufen, Germany) to dryness. The residues were dissolved in $\mathrm{MeOH} / \mathrm{H}_{2} \mathrm{O}(60: 40, v / v)$ solution, filtered, and analyzed using UHPLC—LTQ Orbitrap MS.

Solid-phase extraction (SPE) was used in order to separate anthocyanins from wine sample and grape skin extracts. C18 Sep-Pak cartridges (Phenomenex, Torrance, CA, USA) were preconditioned with $3 \mathrm{~mL}$ of acidified $(0.1 \% \mathrm{HCl})$ methanol (HPLC grade) and $9 \mathrm{~mL}$ of ultrapure water. A total of $2 \mathrm{~mL}$ of sample (previously prepared skin extract and wine) was passed through cartridge, and then the cartridge was washed with $6 \mathrm{~mL}$ of ultrapure water to remove all remain sugars and other polar constituents. The adsorbed anthocyanins were eluted with acidified methanol (1 mL). Prior to UHPLC—LTQ Orbitrap MS analysis, the prepared extracts were filtered through a $0.45 \mu \mathrm{m}$ nylon membrane filter.

\subsection{Spectrophotometric Determination of Total Phenolic Content (TPC), Radical Scavenging Activity (RSA), and Total Anthocyanin Content (TAC)}

A Cintra 6 UV-VIS spectrometer (GBC Scientific Equipment Ltd., Hampshire, IL, USA) was used in all spectrophotometric measurements. TPC was determined using Folin-Ciocalteu reagent, while RSA was measured using DPPH reagent (procedures were described in Pantelić et al. [13]). Gallic acid was used as a standard for total phenolic determination, while the calibration curve for antioxidant measurements was obtained using Trolox. TPC values were expressed as g gallic acid equivalent (GAE) per kg of frozen (skins and pulps) and dried weight (seeds and leaves), as well as g GAE per L of wine. The results for RSA were expressed as mmol of Trolox equivalents (TE) per $\mathrm{kg}$ of frozen (skins and pulps) and dried (seeds and leaves) sample, or as mmol TE per $\mathrm{L}$ of wine. The pH-differential method, described by Pantelić et al. [15], was used in order to define TAC in grape skins and wine. For the purposes of this measurements, buffers of $\mathrm{pH} 1.0(\mathrm{KCl}, 0.025 \mathrm{~mol} / \mathrm{L})$ and $\mathrm{pH} 4.5(\mathrm{NaOAc} / \mathrm{HOAc}, 0.4 \mathrm{~mol} / \mathrm{L})$ were used. The results obtained for TAC were expressed as $\mathrm{g}$ malvidin-3-glucoside (mal-3-glu) equivalents per $\mathrm{kg}$ of frozen grape skin weight and per $\mathrm{L}$ of wine, respectively. All measurements were performed in triplicate, and expressed as mean values \pm standard deviation (SD).

\subsection{UHPLC Orbitrap MS Analysis}

All experiments were performed using a Thermo Scientific ultra-high performance liquid chromatography (UHPLC) system consisting of a quaternary Accela 600 pump and Accela Autosampler, connected to a linear ion trap-orbitrap (LTQ Orbitrap XL) hybrid mass spectrometer with heated-electrospray ionization probe (HESI-II, ThermoFisher Scientific, Bremen, Germany).

Separations were performed on a Syncronis C18-column $(100 \times 2.1 \mathrm{~mm}, 1.7 \mu \mathrm{m}$ particle size $)$ from Thermo Fisher Scientific. The same composition of the mobile phase and the gradient program were used for identification of non-anthocyanins in negative ionization mode and anthocyanins in positive ionization mode. The mobile phase consisted of (A) water $+0.1 \%$ formic acid and (B) acetonitrile + $0.1 \%$ formic acid. A linear gradient program was as follows: $5 \% \mathrm{~B}$ in the first $1.0 \mathrm{~min}, 1.0-14.0 \mathrm{~min}$ $5-95 \% \mathrm{~B}, 14.0-14.2 \mathrm{~min}$ from $95 \%$ to $5 \% \mathrm{~B}$, and $5 \% \mathrm{~B}$ until the $20 \mathrm{~min}$. The injection volume was $5 \mu \mathrm{L}$ and flow rate was $0.3 \mathrm{~mL} \mathrm{~min}^{-1}$. Settings of dynamic exclusion and other ion source parameters were as previously described by Pešić et al. [16]. The normalized collision energy of the collision induced dissociation (CID) cell was set at $35 \mathrm{eV}$. The MS spectra were acquired by full range acquisition covering the $m / z$ range $100-1500$. 
Compounds were identified in the samples according to their mass spectra, exact mass, characteristic fragmentation, and characteristic retention time. Xcalibur software (version 2.1, Thermo Fisher Scientific, Waltham, MA, USA) was used for instrument control, data acquisition and data analysis. The molecule editor program, ChemDraw (version 12.0, CambridgeSoft, Cambridge, MA, USA), was used as a reference library to calculate the exact (monoisotopic) masses of compounds of interest. The data-dependent $\mathrm{MS}^{2}$ events were always performed on the most intense ions detected in the full scan MS. Full scan analysis was employed to detect the monoisotopic mass of unknown compounds, while the fragmentation pathway was obtained by MS ${ }^{4}$.

\section{Results and Discussion}

\subsection{TPC, RSA, and TAC}

According to the results obtained for TPC in grape berries (Table 1), it can be seen that these phytochemicals were most abundant in grape seeds $(51.72-81.07 \mathrm{~g} \mathrm{GAE} / \mathrm{kg})$, followed by grape skins (7.95-14.01 g GAE/kg), while the lowest concentrations of polyphenols were found in the pulps $(0.28-0.82 \mathrm{~g} \mathrm{GAE} / \mathrm{kg})$. Obtained results are in accordance with those published by Pantelić et al. [13], where polyphenolic profiles of seven red grapevine varieties from Serbia were analyzed. The same trends were also obtained for RSA values. The highest antioxidant activity was found in grape seeds (478.05-740.25 mmol TE/kg), followed by the grape skins $(52.15-88.19 \mathrm{mmol} \mathrm{TE} / \mathrm{kg})$ and pulps (11.48-12.30 mmol TE/kg). RSA and TPC values were compared using correlation analysis (MS Excel, Microsoft Office 2007 Professional) and a statistically strong linear relationship was found between them $(r=0.99)$.

Regarding the wine sample, TPC and antioxidant activity were $2.36 \mathrm{~g} \mathrm{GAE} / \mathrm{L}$ and $12.61 \mathrm{mmol}$ $\mathrm{TE} / \mathrm{L}$, respectively. The result obtained for TPC in wine obtained herein was in agreement with those reported for Montenegrin 'Vranac' in a previous publication [17], but somewhat higher compared to the result reported by Radovanović et al. [18] and Matić et al. [19], and lower than those published by Radovanović et al. [20] and Mitić et al. [21]. Mitić et al. [21] examined 'Vranac' wines with different geographical origins, and the values obtained for RSA ranging from 13.00 to $15.02 \mathrm{mmol} \mathrm{TE} / \mathrm{L}$. The result of the current study was within this range.

As for the grape leaves (Table 1), TPC was in the range from 28.98 to $44.01 \mathrm{~g} \mathrm{GAE} / \mathrm{kg}$, while RSA values ranged between 141.77 and $241.39 \mathrm{mmol} \mathrm{TE} / \mathrm{kg}$. Values for TPC in leaves sample are in agreement with the one reported in a previous publication [14].

TAC was determined in grape skins and wine. The obtained results indicated that TAC varied little among the examined skin samples (the average value was $5.73 \mathrm{~g}$ mal 3-glu/ $\mathrm{kg}$ ). In wine sample, TAC was $0.13 \mathrm{~g}$ mal 3-glu/L, which is in accordance with data presented by Pantelić et al. [22].

\subsection{Polyphenolic Profiles of Vranac Leaves, Grapes and Wine}

For the detailed LC/MS characterization of phenolic phytochemicals in the Vranac grapevine variety, one grape sample (seed, pulp, and skin), one leaf sample, and a sample of Vranac wine were selected (sample T1B4 Aerodrom). UHPLC-Orbitrap MS 4 analysis of Vranac leaf, grape, and wine resulted in the detection of 91 compounds in negative ionization mode (Table 2) and 38 in positive ionization mode (Table 3).

The identified compounds represented five structurally distinct groups: (I) phenolic acids and their derivatives (42 compounds); (II) flavan-3-ols (23 compounds); (III) flavanols ( 21 compounds); (IV) stilbenes ( 5 compounds), and (V) anthocyanins (38 compounds). Among all identified compounds, twenty nine were confirmed using standards, while the others were identified by exact mass search of their deprotonated molecule $[\mathrm{M}-\mathrm{H}]^{-}, \mathrm{MS}^{2}, \mathrm{MS}^{3}$, and $\mathrm{MS}^{4}$ fragmentation behavior, as well as by comparison with the available literature $[16,23,24]$. The peak numbers, retention times $\left(t_{R}, \mathrm{~min}\right)$, compound names, molecular formulas, calculated and exact masses $\left([\mathrm{M}-\mathrm{H}]^{-} / \mathrm{M}^{+}, m / z\right)$, mean mass accuracy errors (ppm), major $\mathrm{MS}^{2}, \mathrm{MS}^{3}$, and $\mathrm{MS}^{4}$ fragment ions $(\mathrm{m} / \mathrm{z})$, as well as presence of selected compound in investigated extracts are summarized in Tables 2 and 3. Anthocyanins were analyzed only in the Vranac wine and grape berry skin extract. 


\subsubsection{Phenolic Acids and Their Derivatives}

Phenolic acids (hydroxycinnamic and hydroxybenzoic acid derivatives), commonly present in grape and wines [25], were found as free and in the form of glycosides (pentoside and hexoside derivatives) and esters with tartaric, treonic, shikimic and quinic acids. Specifically, 32 phenolic acids and their derivatives were found in analyzed wine and leaf samples, while in seed, skin, and pulp a total of 20,11, and 9 (respectively) compounds were identified. Protocatechuic acid (compound 3), caffeoyltartaric (caftaric) acid (compound 4), hydroxybenzoic acid hexoside (compound 6), coumaryltartaric (coutaric) acid (compound 11) and vanillic acid (compound 28) were identified in all tested samples (Table 2). Compounds 7, 9, $\mathbf{1 4}$ and $\mathbf{3 0}$ were identified only in leaf samples, while compounds 12, 18, 22 and 24 were identified in wine samples only. Chlorogenic acid, as a hydroxycinnamic acid that was detected in leaves sample only, in previous studies has been associated with the reduction of oxidative stress conditions [26].

Presence and the content of ellagic acid (35) in various parts of the plant issue have attracted the attention of researchers. Ellagic acid is considered as a compound that exhibits a wide range of biological activities and could be beneficial for human health. In this research, ellagic acid pentoside (22) was detected only in leaves extracts, which supports the claims that the grape leaf extract has high nutritional value and can be used as supplements in human diet [14].

Two isomeric coumaryltartaric acids, 11 and 17, with identical molecular ion $\left([\mathrm{M}-\mathrm{H}]^{-}\right.$at $\left.295 \mathrm{~m} / \mathrm{z}\right)$, but showing slightly different MS fragmentation patterns, were identified at 4.95 and $5.36 \mathrm{~min}$, respectively. Compound 11 generated MS $^{2}$ base peak at $163 \mathrm{~m} / \mathrm{z}$ (mass of deprotonated coumaric acid), while compound 17 gave MS$^{2}$ base peak at $149 \mathrm{~m} / \mathrm{z}$ (mass of deprotonated tartaric acid). In addition, feruoyltartaric (fertaric) acid (compound 21) and caffeoyltreonic acid (compound 26) were identified in wine and leaf samples. Compound 29 at $5.99 \mathrm{~min}$ and $335 \mathrm{~m} / \mathrm{z}$ giving MS ${ }^{2}$ base peak at $179 \mathrm{~m} / \mathrm{z}$ (corresponding to deprotonated caffeic acid) and $\mathrm{MS}^{2}$ secondary peak at $135 \mathrm{~m} / \mathrm{z}$ was identified as caffeoylshikimic acid. Compounds $\mathbf{1}$ and 18, with same accurate masses $(315 \mathrm{~m} / \mathrm{z})$ and similar fragmentation patterns, were marked as dihydroxybenzoic acid hexoside isomers. The first isomer at $2.89 \mathrm{~min}$ was identified only in grape berry (skin, pulp, and skin), while the second was identified only in wine.

\subsubsection{Flavan-3-ols}

From the group flavan-3-ols, all identified compound (monomers, dimers, trimers) were found in grape seed samples and their identification was largely based on the evaluated MS fragments and previously reported data about phytochemicals found in wine and grapes [27]. Presence of compounds 47 (gallocatechin), 54 (catechin), 56 (epigallocatechin), 60 (epicatechin), and 62 (catechin 3-O-gallate) were confirmed using available standards.

In the seeds, as the richest source of flavan-3-ol, twenty three compounds were identified. The skin

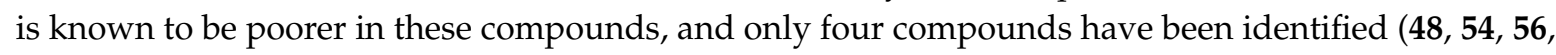
and 60). Of all the identified flavan-3-ol from the seed, seventeen flavan-3-ols were also identified in the wine sample. A match in flavan-3-ols between samples of seed and wine was also expected, given the extraction of phenolic compounds from the seed into the wines during the maceration process. Unlike flavan-3-ols from the seed, all flavan-3-ols detected in the skin samples (total of four compounds) were also identified in the wine.

The results of the examination of phenolic composition of leaves of Vranac grapevine variety and another nine red wine varieties from different Serbian wine-growing regions indicate the variation in the profile of flavan-3-ols in leaves of different varieties [14].

Compounds $43(4.14 \mathrm{~min}$ and $451 \mathrm{~m} / \mathrm{z})$ and $46(4.81 \mathrm{~min}$ and $451 \mathrm{~m} / \mathrm{z})$ with the same fragmentation pathway were identified only in grape seed, as (epi)catechin hexoside isomers. Both compounds generated MS ${ }^{2}$ base peak at $289 \mathrm{~m} / \mathrm{z}$, corresponding to deprotonated catechin or epicatechin. It is interesting to note that some compounds that are not common for Vitis species, such as chalcan-flavan dimers, were found in wine and grape seed samples. Two isomers of chalcan-flavan-3-ol (compounds 
51 and 58, molecular ion at $579 \mathrm{~m} / \mathrm{z}$ ) with same fragmentation pathway were identified at 5.07 and $5.59 \mathrm{~min}$, respectively, and their fragmentation was in accordance with literature data [28]. Compound 65 at $8.12 \mathrm{~min}$ and molecular ion $435 \mathrm{~m} / \mathrm{z}$ was marked as (epi)catechin 3-O-coumarate. It produced MS $^{2}$ base peak at $289 \mathrm{~m} / \mathrm{z}$ and further fragmentation confirmed that this was an ester of catechin and coumaric acid (Figure 1). These compounds were already isolated and identified in green tea extracts [29], and as far as we know, they have not been identified in some Vitis species until now. In this study, it was found only in grape seeds extract of the autochthonous grapevine variety Vranac. A very similar fragmentation pattern was noticed for (epi)catechin 3-O-vanillate (64) and it was recently identified in grape seed and red wine by Ma et al. [30].

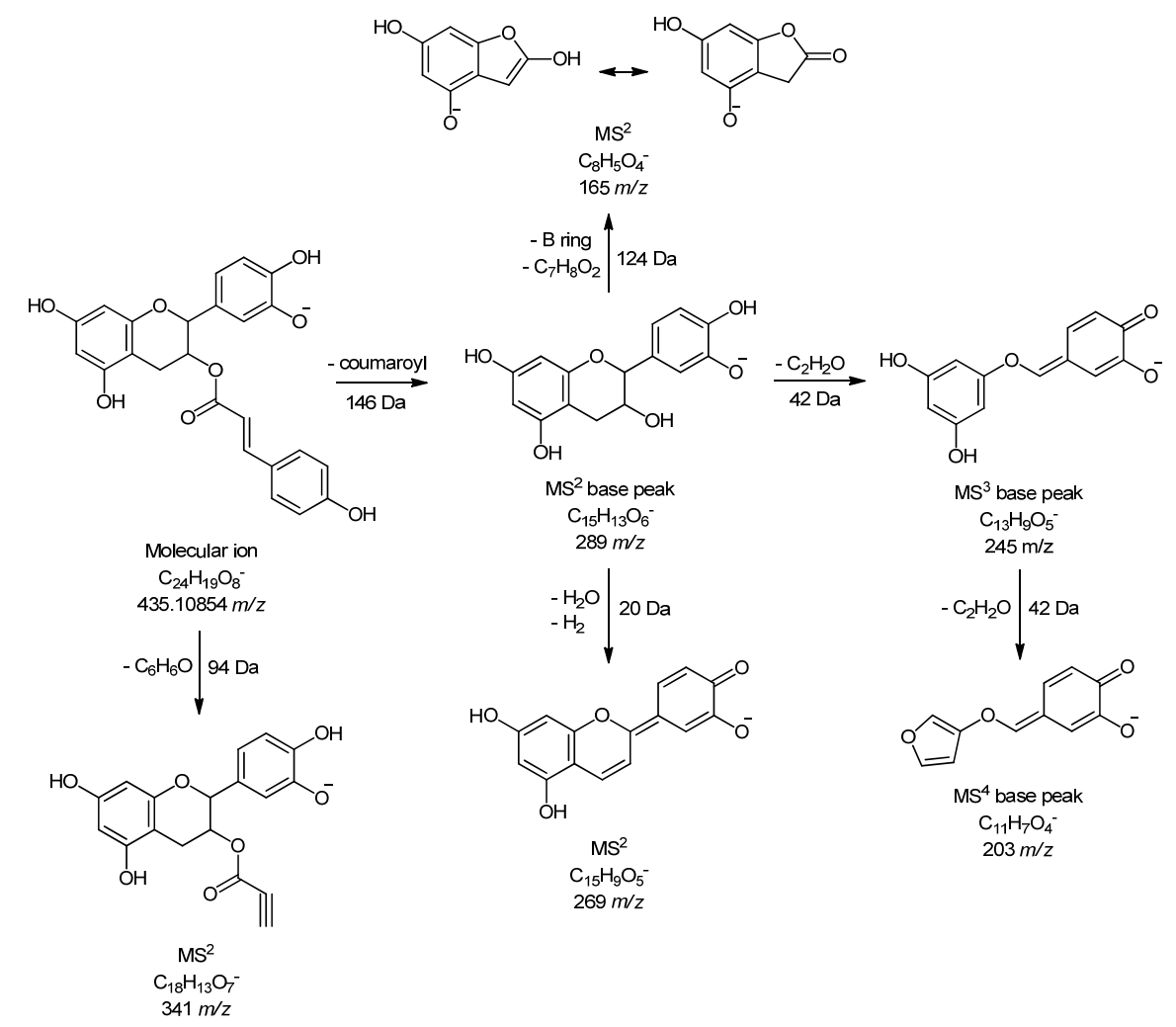

Figure 1. Fragmentation pathway of (Epi)catechin 3-O-coumarate (65) in negative ionization mode.

\subsubsection{Flavanols}

Among twenty one flavanols, six were identified using available standards (myricetin -67 , quercetin 3-O-(6"rhamnosyl)glucoside-70, quercetin 3-O-galactoside-73, kaempferol 3-O-glucoside -76, quercetin-85, and kaempferol-86). Compound 73 (quercetin 3-O-galactoside) and compound 79 (quercetin 3-O-hexuronide) were identified in all investigated samples. It is well known that various derivatives of flavanol aglycones and glycosides are commonly present in grapes and wines [25]. For example, syringetin 3-O-hexoside (compound 77), eluted at 6.99 min with molecular ion at $507 \mathrm{~m} / \mathrm{z}$, was confirmed by examination of its MS data. It is well known that this compound is specific to Vitis species because it was previously identified in grape samples [31]. Compound 81 (quercetin 3-O-(6"-malonyl)hexoside), found only in Vranac leaf sample at $7.36 \mathrm{~min}$ and $549 \mathrm{~m} / \mathrm{z}$, gave $\mathrm{MS}^{2}$ base peak at $505 \mathrm{~m} / \mathrm{z}$ (loss of $\left.\mathrm{CO}_{2}-44 \mathrm{Da}\right)$ and $\mathrm{MS}^{3}$ and $\mathrm{MS}^{4}$ spectrum which corresponded to the fragmentation of quercetin.

A large number of compounds from the flavanol group were present in the skin sample. Of the 21 identified flavanols, 14 were present in the skin while 4 compounds were present in the seeds $(73,79$, 78 , and 85). 
According to previous research, quercetin and kaempferol derivatives are the main leaf flavonoids found in Vitis vinifera species [32] and our research also supports this knowledge. Of the total number of detected flavanols (a total of 21 compounds), only two (77 and 78) were not detected in the leaves, and five compounds $(\mathbf{7 4}, \mathbf{7 5}, \mathbf{8 1}, \mathbf{8 3}$, and $\mathbf{8 4})$ were present only in leaves. None of the flavanol derivatives that were detected solely in the leaves were detected in the grape skin sample. It is believed that the grape leaves are rich in polyphenols, specifically flavonoids, since these compounds exhibit UV protective effects and can protect chloroplast from the damaging effects of UV rays [33].

The presence of kaempferol 3-O-hexuronide methyl ether and kaempferol 7-O-(6-malonyl)hexoside (83 and 84) in the leaves samples of Vranac grown in Montenegro (Mediterranean climate) is contrary to the results from the Central Serbia region, were kaempferol compounds were not identified in Vranac leaves [14]. These discrepancies may be due to different climatic conditions between growing regions that influence the synthesis of these compounds.

\subsubsection{Stilbenes}

As for stilbene derivatives, aside from trans-resveratrol, confirmed using available standard, four other derivatives were identified using exact mass search and MS fragmentation. Compound $\mathbf{8 7}$ with retention time $7.11 \mathrm{~min}$ and $389 \mathrm{~m} / \mathrm{z}$ was identified as resveratrol hexoside. It generated $\mathrm{MS}^{2}$ base peak at $277 \mathrm{~m} / \mathrm{z}$, which was obtained by the loss of one sugar unit (hexosyl residue-162 Da). MS 3 base peak at $227 \mathrm{~m} / \mathrm{z}$ confirmed the presence of resveratrol as aglycone. Polymeric stilbene derivatives are common for the genus Vitis [34] and in our samples of grape seeds and wine it was possible to identify resveratrol dimer (compound 90, $453 \mathrm{~m} / \mathrm{z}$ ), trimer (compound 91, $679 \mathrm{~m} / \mathrm{z}$ ) and tetramer (compound 89, $905 \mathrm{~m} / \mathrm{z})$.

\subsubsection{Anthocyanins}

Monomeric anthocyanins derived from grape skin are of the highest importance for the color of young red wines. Consequently, the composition of free anthocyanins in grapes determines the composition of the anthocyanin profile of the produced red wines to a significant extent [35].

The most abundant compounds found in the Vitis vinifera red wines are malvidin derivatives and the main monomeric anthocyanins are the 3-O-monoglucosides. The largest number of compounds found in Vranac wine and berry skin extracts are malvidin derivatives (19 compounds), followed by peonidin ( 7 compounds) and delphinidin ( 6 compounds) derivatives. It is known that among monomeric anthocyanins, malvidin 3-O-glucoside and its derivatives are usually the most abundant and are the main source of the red color in very young red wines [35].

The presence of diglucoside anthocyanins in non- $V$. vinifera grape and wine is common, both 3-O-monoglucoside and 3,5-O-diglucoside of anthocyanins can be present [13]. Diglucosides are more stable than their monoglucoside derivatives but are more susceptible to browning and are less colored [36]. Some previous investigations have detected the presence of 3,5-O-diglucosides in grape or wine of $V$. vinifera varieties [13]. We also identified peonidine 3,5-di-O-hexoside (1') in the Vranac skin sample. It represented the major diglucoside in wine from non- $V$. vinifera grapes. The same diglucoside has not been identified in Vranac wine. In addition, malvidin 3,5-di-O-glucoside (14') was also identified in the skin and wine sample.

In addition, several acylated anthocyanin derivatives were found in investigated extracts. For example, malvidin 3-O-(6"-caffeoyl)hexoside (22'), found at $6.39 \mathrm{~min}$ and $655 \mathrm{~m} / \mathrm{z}$, showed $\mathrm{MS}^{2}$ base peak at $331 \mathrm{~m} / \mathrm{z}$ (deprotonated malvidin), corresponding to loss of caffeoylhexoside residue (324 Da). Further loss of $16 \mathrm{Da}\left(\mathrm{CH}_{4}\right)$ and $28 \mathrm{Da}(\mathrm{CO})$ gave $\mathrm{MS}^{3}$ and $\mathrm{MS}^{4}$ base peaks at 315 and $287 \mathrm{~m} / \mathrm{z}$, respectively.

All peonidin derivatives (except peonidin 3-O-hexoside-pyruvate, 4', Figure 2A) exhibit a specific fragment ion at $301 \mathrm{~m} / \mathrm{z}$, corresponding to aglycone part of molecule (Table 3). 
Table 1. Total phenolic contents (TPC, g GAE/kg) and radical scavenging activities (RSA, mmol TE/kg) in the skins, seeds, pulps, and leaves of grapevine samples. Total anthocyanin content (TAC, g mal 3-glu/kg) in grape skins.

\begin{tabular}{|c|c|c|c|c|c|c|c|c|c|c|}
\hline \multicolumn{2}{|c|}{ Samples } & T1B4 Aerodrom & T2B4 Aerodrom & T3B4 Aerodrom & T4B4 Aerodrom & T1B2 Bunar 12 & T2B2 Bunar 12 & T10 Šipčanik & T11 Šipčanik & T12 Šipčanik \\
\hline \multirow{3}{*}{ Skin (S) } & TPC & $8.76 \pm 0.12$ & $7.95 \pm 0.08$ & $9.77 \pm 0.09$ & $11.34 \pm 0.22$ & $12.06 \pm 0.08$ & $10.80 \pm 0.09$ & $11.75 \pm 0.11$ & $14.01 \pm 0.01$ & $11.13 \pm 0.30$ \\
\hline & RSA & & & & & & & 77.41 & $88.19 \pm$ & $71.32 \pm 1.28$ \\
\hline & TAC & $5.47 \pm 0.01$ & $4.43 \pm 0.01$ & $4.26 \pm 0.00$ & $6.48 \pm 0.02$ & $7.44 \pm 0.01$ & $6.02 \pm 0.05$ & $4.95 \pm 0.04$ & $7.58 \pm 0.00$ & $4.91 \pm 0.01$ \\
\hline \multirow{2}{*}{ Pulp (P) } & ТPC & $82+007$ & $33 \pm 0.01$ & $28 \pm 0.00$ & $51 \pm 0.00$ & $0.33 \pm 0.02$ & $0.35 \pm 0.03$ & $0.36 \pm 0.01$ & $0.42 \pm 0.01$ & $0.40 \pm 0.00$ \\
\hline & RSA & $11.66 \pm 0.25$ & $11.97 \pm 0.30$ & $11.48 \pm 0.49$ & $12.27 \pm 0.03$ & $11.70 \pm 0.05$ & $11.82 \pm 0.33$ & $12.29 \pm 0.00$ & $12.30 \pm 0.03$ & $12.23 \pm 0.10$ \\
\hline \multirow{2}{*}{ Seed (E) } & TPC & $57.54 \pm 0.91$ & $7058+058$ & $7046+$ & $61.77 \pm 0.08$ & $51.72 \pm 0.83$ & $66.23 \pm 2.24$ & $72.20 \pm 0.32$ & $73.57 \pm 0.66$ & $81.07 \pm 0.40$ \\
\hline & RSA & $613.24 \pm 17.60$ & $655.96 \pm 3.27$ & $617.84 \pm 1.63$ & $571.63 \pm 27.77$ & $478.05 \pm 3.27$ & $673.19 \pm 6.40$ & $740.25 \pm 15.24$ & $716.18 \pm 12.80$ & $634.61 \pm 25.38$ \\
\hline \multirow{2}{*}{ Leaf (L) } & TPC & $40.30 \pm 0.21$ & $44.01 \pm 0.86$ & $41.15 \pm 0.33$ & $33.78 \pm 0.85$ & $28.98 \pm 0.61$ & $29.44 \pm 0.56$ & 31.96 & $32.46 \pm 0.17$ & $35.44 \pm 1.01$ \\
\hline & RSA & $201.21 \pm 2.65$ & $219.74 \pm 4.68$ & $241.39 \pm 11.68$ & $170.44 \pm 6.58$ & $163.72 \pm 8.77$ & $141.77 \pm 6.07$ & $190.92 \pm 7.90$ & $200.37 \pm 0.00$ & $185.77 \pm 11.25$ \\
\hline
\end{tabular}

T-table; B-block; Aerodrom, Bunar 12, and Šipčanik—vineyards located at the "Ćemovsko" field (Montenegro).

Table 2. High resolution MS data and negative ion $\mathrm{MS}^{2}, \mathrm{MS}^{3}$, and $\mathrm{MS}^{4}$ fragmentation of phenolic compounds identified in Vranac wine (W), grape (seed (S), pulp (P), and skin (E)) and leaf (L) extracts.

\begin{tabular}{|c|c|c|c|c|c|c|c|c|c|c|c|c|c|c|}
\hline No & $t_{\mathrm{R}}, \min$ & Compound Name & $\begin{array}{c}\text { Molecular } \\
\text { Formula, } \\
{[\mathrm{M}-\mathrm{H}]^{-}}\end{array}$ & $\begin{array}{c}\text { Calculated } \\
\text { Mass, } \\
{[\mathrm{M}-\mathrm{H}]^{-}}\end{array}$ & $\begin{array}{c}\text { Exact } \\
\text { Mass, } \\
{[\mathrm{M}-\mathrm{H}]^{-}}\end{array}$ & $\Delta \mathrm{ppm}$ & $\begin{array}{c}\text { MS }^{2} \\
\text { Fragments, } \\
\text { (\% Base } \\
\text { Peak) }\end{array}$ & $\begin{array}{c}\text { MS }^{3} \\
\text { Fragments, } \\
\text { (\% Base } \\
\text { Peak) }\end{array}$ & $\begin{array}{c}\text { MS }^{4} \\
\text { Fragments, } \\
\text { (\% Base } \\
\text { Peak) }\end{array}$ & $\mathbf{W}$ & $\mathrm{S}$ & $\mathbf{P}$ & E & L \\
\hline \multicolumn{15}{|c|}{ Phenolic Acids and Their Derivatives } \\
\hline 1 & 2.89 & $\begin{array}{l}\text { Dihydroxybenzoic acid } \\
\text { hexoside isomer } 1\end{array}$ & $\mathrm{C}_{13} \mathrm{H}_{15} \mathrm{O}_{9}^{-}$ & 315.07216 & 315.07135 & 2.57 & $\begin{array}{c}153(100), 152 \\
(50), 109(15) \\
108(10)\end{array}$ & $109(100)$ & $\begin{array}{c}123(25), 109 \\
(10), 85(10) \\
81(100)\end{array}$ & - & + & + & + & - \\
\hline 2 & 3.01 & Gallic acid $^{a}$ & $\mathrm{C}_{7} \mathrm{H}_{5} \mathrm{O}_{5}^{-}$ & 169.01425 & 169.01369 & 3.31 & $125(100)$ & $107(100)$ & - & - & + & - & - & + \\
\hline 3 & 3.86 & Protocatechuic acid $^{a}$ & $\mathrm{C}_{7} \mathrm{H}_{5} \mathrm{O}_{4}^{-}$ & 153.01933 & 153.01906 & 1.76 & $\begin{array}{c}109(100), 95 \\
(75), 79(20) \\
59(10)\end{array}$ & $\begin{array}{l}81(100), 68 \\
(25), 65(15)\end{array}$ & - & + & + & + & + & + \\
\hline 4 & 4.20 & Caffeoyltartaric acid & $\mathrm{C}_{13} \mathrm{H}_{11} \mathrm{O}_{9}^{-}$ & 311.04031 & 311.04001 & 0.97 & $\begin{array}{c}179(40), 177 \\
(15), \mathbf{1 4 9}(100)\end{array}$ & $\begin{array}{c}131(50), 103 \\
(90), 87(100), \\
59(25)\end{array}$ & $59(100)$ & + & + & + & + & + \\
\hline 5 & 4.60 & Gallic acid hexoside & $\mathrm{C}_{13} \mathrm{H}_{15} \mathrm{O}_{10}^{-}$ & 331.06707 & 331.06638 & 2.08 & $\begin{array}{c}169 \text { (100), } 125 \\
(5)\end{array}$ & $125(100)$ & $\begin{array}{c}110(10), 97 \\
(30), 81(100) \\
53(30)\end{array}$ & + & + & + & - & + \\
\hline
\end{tabular}


Table 2. Cont.

\begin{tabular}{|c|c|c|c|c|c|c|c|c|c|c|c|c|c|c|}
\hline No & $t_{\mathrm{R}}, \min$ & Compound Name & $\begin{array}{l}\text { Molecular } \\
\text { Formula, } \\
{[\mathrm{M}-\mathrm{H}]^{-}}\end{array}$ & $\begin{array}{l}\text { Calculated } \\
\text { Mass, } \\
{[\mathrm{M}-\mathrm{H}]^{-}}\end{array}$ & $\begin{array}{r}\text { Exact } \\
\text { Mass, } \\
{[\mathrm{M}-\mathrm{H}]^{-}}\end{array}$ & $\Delta \mathrm{ppm}$ & $\begin{array}{c}\text { MS }^{2} \\
\text { Fragments, } \\
\text { (\% Base } \\
\text { Peak) }\end{array}$ & $\begin{array}{c}\text { MS }^{3} \\
\text { Fragments, } \\
\text { (\% Base } \\
\text { Peak) }\end{array}$ & $\begin{array}{c}\text { MS }^{4} \\
\text { Fragments, } \\
\text { (\% Base } \\
\text { Peak) }\end{array}$ & $\mathbf{W}$ & $\mathbf{S}$ & $\mathbf{P}$ & $\mathbf{E}$ & L \\
\hline 6 & 4.62 & $\begin{array}{l}\text { Hydroxybenzoic acid } \\
\text { hexoside isomer } 1\end{array}$ & $\mathrm{C}_{13} \mathrm{H}_{15} \mathrm{O}_{8}^{-}$ & 299.07724 & 299.07648 & 2.54 & $137(100)$ & $93(100)$ & - & + & + & + & + & + \\
\hline 7 & 4.72 & Chlorogenic acid hexoside & $\mathrm{C}_{22} \mathrm{H}_{27} \mathrm{O}_{14}^{-}$ & 515.14008 & 515.13917 & 1.77 & $\begin{array}{c}353(40), 341 \\
(100), 335(25), \\
191(15), 179 \\
(45)\end{array}$ & $\begin{array}{c}179(100), 135 \\
(10)\end{array}$ & $135(100)$ & - & - & - & - & + \\
\hline 8 & 4.81 & Caffeic acid hexoside & $\mathrm{C}_{15} \mathrm{H}_{17} \mathrm{O}_{9}^{-}$ & 341.08781 & 341.08659 & 3.58 & $\begin{array}{c}191(10), 179 \\
(100), 135(10)\end{array}$ & $135(100)$ & $\begin{array}{c}135(100), 107 \\
(50)\end{array}$ & + & - & - & - & + \\
\hline 9 & 4.84 & $\begin{array}{c}\text { Dihydroxybenzoic acid } \\
\text { hexosyl pentoside isomer } 1\end{array}$ & $\mathrm{C}_{18} \mathrm{H}_{23} \mathrm{O}_{13}-$ & 447.11441 & 447.11353 & 1.97 & $\begin{array}{l}315(100), 285 \\
(10), 153(10)\end{array}$ & $\begin{array}{c}153(100), 123 \\
(10)\end{array}$ & $123(100)$ & - & - & - & - & + \\
\hline 10 & 4.90 & Chlorogenic acid $^{a}$ & $\mathrm{C}_{16} \mathrm{H}_{17} \mathrm{O}_{9}^{-}$ & 353.08781 & 353.08633 & 4.19 & $\begin{array}{l}191(100), 179 \\
(30), 135(10)\end{array}$ & $\begin{array}{l}173(75), 127 \\
(100), 111(40), \\
93(60), 85(90)\end{array}$ & $\begin{array}{c}109(30), 99 \\
(40), 85(100)\end{array}$ & + & + & - & - & + \\
\hline 11 & 4.95 & $\begin{array}{c}\text { Coumaroyltartaric acid } \\
\text { isomer } 1\end{array}$ & $\mathrm{C}_{13} \mathrm{H}_{11} \mathrm{O}_{8}^{-}$ & 295.04594 & 295.04532 & 2.10 & $\begin{array}{c}163(100), 149 \\
(10), 119(5)\end{array}$ & $119(100)$ & $\begin{array}{l}119(100), 93 \\
\quad(50)\end{array}$ & + & + & + & + & + \\
\hline 12 & 4.96 & Gallic acid methyl ester & $\mathrm{C}_{8} \mathrm{H}_{7} \mathrm{O}_{5}^{-}$ & 183.02990 & 183.02931 & 3.22 & $\begin{array}{c}168(100), 124 \\
(15)\end{array}$ & $124(100)$ & $95(100)$ & + & - & - & - & - \\
\hline 13 & 5.01 & $\begin{array}{l}\text { Hydroxybenzoic acid } \\
\text { hexoside isomer } 2\end{array}$ & $\mathrm{C}_{13} \mathrm{H}_{15} \mathrm{O}_{8}^{-}$ & 299.07724 & 299.07593 & 4.38 & $137(100)$ & $93(100)$ & - & + & - & - & - & + \\
\hline 14 & 5.04 & $\begin{array}{c}\text { Dihydroxybenzoic acid } \\
\text { hexosyl pentoside isomer } 2\end{array}$ & $\mathrm{C}_{18} \mathrm{H}_{23} \mathrm{O}_{13}{ }^{-}$ & 447.11441 & 447.11356 & 1.90 & $\begin{array}{c}315(100), 285 \\
(60), 153(50) \\
149(25)\end{array}$ & $\begin{array}{c}153(100), 123 \\
(10)\end{array}$ & $\begin{array}{c}123(100), 109 \\
\text { (5) }\end{array}$ & - & - & - & - & + \\
\hline 15 & 5.26 & $p$-Hydroxybenzoic acid ${ }^{a}$ & $\mathrm{C}_{7} \mathrm{H}_{5} \mathrm{O}_{3}^{-}$ & 137.02442 & 137.02385 & 4.16 & $\begin{array}{c}109(10), 93 \\
(100)\end{array}$ & $93(100)$ & - & + & + & + & - & + \\
\hline 16 & 5.32 & $\begin{array}{l}\text { Coumaric acid hexoside } \\
\text { isomer } 1\end{array}$ & $\mathrm{C}_{15} \mathrm{H}_{17} \mathrm{O}_{8}^{-}$ & 325.09289 & 325.09167 & 3.75 & $\begin{array}{c}163(100), 119 \\
(10)\end{array}$ & $119(100)$ & - & + & - & - & + & + \\
\hline 17 & 5.36 & $\begin{array}{c}\text { Coumaroyltartaric acid } \\
\text { isomer } 2\end{array}$ & $\mathrm{C}_{13} \mathrm{H}_{11} \mathrm{O}_{8}^{-}$ & 295.04594 & 295.04501 & 3.15 & $\begin{array}{l}163(30), \mathbf{1 4 9} \\
(100), 119(5)\end{array}$ & $\begin{array}{c}131(50), 103 \\
(90), 87(100) \\
59(30)\end{array}$ & - & + & - & - & - & + \\
\hline 18 & 5.44 & $\begin{array}{l}\text { Dihydroxybenzoic acid } \\
\text { hexoside isomer } 2\end{array}$ & $\mathrm{C}_{13} \mathrm{H}_{15} \mathrm{O}_{9}^{-}$ & 315.07216 & 315.07123 & 2.95 & $\begin{array}{l}153(100), 135 \\
(10), 109(10)\end{array}$ & $\begin{array}{c}135(100), 109 \\
(50)\end{array}$ & $91(100)$ & + & - & - & - & - \\
\hline 19 & 5.47 & 3-O-p-Coumaroylquinic acid & $\mathrm{C}_{16} \mathrm{H}_{17} \mathrm{O}_{8}^{-}$ & 337.09289 & 337.09171 & 3.50 & $\begin{array}{c}191(10), 173 \\
(10), 163(100) \\
119(10)\end{array}$ & $119(100)$ & - & + & - & - & - & + \\
\hline
\end{tabular}


Table 2. Cont

\begin{tabular}{|c|c|c|c|c|c|c|c|c|c|c|c|c|c|c|}
\hline No & $t_{\mathrm{R}}, \min$ & Compound Name & $\begin{array}{c}\text { Molecular } \\
\text { Formula, } \\
{[\mathrm{M}-\mathrm{H}]^{-}}\end{array}$ & $\begin{array}{l}\text { Calculated } \\
\text { Mass, } \\
{[\mathrm{M}-\mathrm{H}]^{-}}\end{array}$ & $\begin{array}{r}\text { Exact } \\
\text { Mass, } \\
{[\mathrm{M}-\mathrm{H}]^{-}}\end{array}$ & $\Delta \mathrm{ppm}$ & $\begin{array}{c}\text { MS }^{2} \\
\text { Fragments, } \\
\text { (\% Base } \\
\text { Peak) }\end{array}$ & $\begin{array}{c}\text { MS }^{3} \\
\text { Fragments, } \\
\text { (\% Base } \\
\text { Peak) }\end{array}$ & $\begin{array}{c}\text { MS }^{4} \\
\text { Fragments, } \\
\text { (\% Base } \\
\text { Peak) }\end{array}$ & $\mathbf{W}$ & $\mathrm{S}$ & $\mathbf{P}$ & E & \\
\hline 20 & 5.51 & $\begin{array}{l}\text { Coumaric acid hexoside } \\
\text { isomer } 2\end{array}$ & $\mathrm{C}_{15} \mathrm{H}_{17} \mathrm{O}_{8}^{-}$ & 325.09289 & 325.09164 & 3.85 & $\begin{array}{c}289(20), 265 \\
(20), 187(40) \\
163(80), 145 \\
(100)\end{array}$ & $117(100)$ & - & + & - & - & + & . \\
\hline 21 & 5.62 & Feruloyltartaric acid & $\mathrm{C}_{14} \mathrm{H}_{13} \mathrm{O}_{9}^{-}$ & 325.05651 & 325.05518 & 4.09 & $\begin{array}{c}193(100), 113 \\
(5)\end{array}$ & $\begin{array}{c}178(65), 149 \\
(100), 134(40)\end{array}$ & $134(100)$ & + & - & - & - & \\
\hline 22 & 5.66 & Ferulic acid pentoside & $\mathrm{C}_{15} \mathrm{H}_{17} \mathrm{O}_{8}^{-}$ & 325.09289 & 325.09135 & 4.74 & $193(100)$ & $\begin{array}{l}178(60), 149 \\
(100), 134(30)\end{array}$ & $134(100)$ & + & - & - & - & \\
\hline 23 & 5.66 & Ferulic acid hexoside & $\mathrm{C}_{16} \mathrm{H}_{19} \mathrm{O}_{9}{ }^{-}$ & 355.10346 & 355.10199 & 4.14 & $\begin{array}{c}217(60), 193 \\
(100), 175(40) \\
134(10)\end{array}$ & $\begin{array}{c}178(20), 149 \\
(40), 134(100)\end{array}$ & $\begin{array}{l}134(50), 106 \\
(100)\end{array}$ & + & - & - & - & \\
\hline 24 & 5.67 & $p$-Hydroxyphenylacetic acid & $\mathrm{C}_{8} \mathrm{H}_{7} \mathrm{O}_{3}^{-}$ & 151.04007 & 151.03996 & 0.73 & $\begin{array}{c}136(100), 95 \\
(5)\end{array}$ & $\begin{array}{c}108(25), 92 \\
(100)\end{array}$ & $108(100)$ & + & - & - & - & \\
\hline 25 & 5.67 & Ellagic acid hexoside & $\mathrm{C}_{20} \mathrm{H}_{15} \mathrm{O}_{13}^{-}$ & 463.05181 & 463.05078 & 2.22 & $\begin{array}{c}302(15), 301 \\
(100), 300(50) \\
289(10), 273 \\
(10)\end{array}$ & $\begin{array}{c}301(70), 284 \\
(20), 257(100) \\
229(65), 185 \\
(30)\end{array}$ & $\begin{array}{l}229(50), 213 \\
(30), 185(100)\end{array}$ & - & + & - & - & \\
\hline 26 & 5.82 & Caffeoylthreonic acid & $\mathrm{C}_{13} \mathrm{H}_{13} \mathrm{O}_{8}^{-}$ & 297.06159 & 297.06030 & 4.34 & $\begin{array}{c}179(15), 135 \\
(100)\end{array}$ & $\begin{array}{l}117(100), 89 \\
(90), 75(80)\end{array}$ & $89(100)$ & + & - & - & - & . \\
\hline 27 & 5.84 & Caffeic acid ${ }^{a}$ & $\mathrm{C}_{9} \mathrm{H}_{7} \mathrm{O}_{4}^{-}$ & 179.03498 & 179.03436 & 3.46 & $\begin{array}{c}135(100), 117 \\
(10), 91(20) \\
59(15)\end{array}$ & $\begin{array}{l}107(100), 59 \\
\quad(50)\end{array}$ & - & + & + & - & + & \\
\hline 28 & 5.89 & Vanillic acid $^{a}$ & $\mathrm{C}_{8} \mathrm{H}_{7} \mathrm{O}_{4}^{-}$ & 167.03498 & 167.03433 & 3.89 & $\begin{array}{c}153(10), 152 \\
(80), 124(10) \\
123(100), 108 \\
(20)\end{array}$ & $108(100)$ & $79(100)$ & + & + & + & + & . \\
\hline 29 & 5.99 & Caffeoylshikimic acid & $\mathrm{C}_{16} \mathrm{H}_{15} \mathrm{O}_{8}^{-}$ & 335.07724 & 335.07654 & 2.09 & $\begin{array}{c}179(100), 135 \\
(25)\end{array}$ & $135(100)$ & $107(100)$ & + & + & - & - & . \\
\hline 30 & 6.11 & Ellagic acid pentoside & $\mathrm{C}_{19} \mathrm{H}_{13} \mathrm{O}_{12}{ }^{-}$ & 433.04125 & 433.04080 & 1.04 & $\begin{array}{c}301(100), 300 \\
(80)\end{array}$ & $\begin{array}{c}301(95), 284 \\
(25), 257(100), \\
229(70), 185 \\
(40)\end{array}$ & $\begin{array}{c}229(70), 213 \\
(30), 201(15) \\
185(100)\end{array}$ & - & - & - & - & \\
\hline 31 & 6.20 & Syringic acid ${ }^{a}$ & $\mathrm{C}_{9} \mathrm{H}_{9} \mathrm{O}_{5}^{-}$ & 197.04555 & 197.04474 & 4.11 & $\begin{array}{l}183(100), 153 \\
(40), 138(10)\end{array}$ & $\begin{array}{c}167(100), 138 \\
(10), 123(5)\end{array}$ & - & + & - & + & + & . \\
\hline
\end{tabular}


Table 2. Cont.

\begin{tabular}{|c|c|c|c|c|c|c|c|c|c|c|c|c|c|c|}
\hline No & $t_{\mathrm{R}}, \min$ & Compound Name & $\begin{array}{c}\text { Molecular } \\
\text { Formula, } \\
{[\mathrm{M}-\mathrm{H}]^{-}}\end{array}$ & $\begin{array}{c}\text { Calculated } \\
\text { Mass, } \\
{[\mathrm{M}-\mathrm{H}]^{-}}\end{array}$ & $\begin{array}{c}\text { Exact } \\
\text { Mass, } \\
{[\mathrm{M}-\mathrm{H}]^{-}}\end{array}$ & $\Delta \mathrm{ppm}$ & $\begin{array}{c}\text { MS }^{2} \\
\text { Fragments, } \\
\text { (\% Base } \\
\text { Peak) }\end{array}$ & $\begin{array}{c}\mathrm{MS}^{3} \\
\text { Fragments, } \\
\text { (\% Base } \\
\text { Peak) }\end{array}$ & $\begin{array}{c}\text { MS }^{4} \\
\text { Fragments, } \\
\text { (\% Base } \\
\text { Peak) }\end{array}$ & W & $\mathbf{S}$ & $\mathbf{P}$ & $\mathrm{E}$ & $\mathbf{L}$ \\
\hline 32 & 6.25 & $\begin{array}{c}\text { Coumaric acid hexoside } \\
\text { isomer } 3\end{array}$ & $\mathrm{C}_{15} \mathrm{H}_{17} \mathrm{O}_{8}^{-}$ & 325.09289 & 325.09180 & 3.35 & $\begin{array}{c}289(10), 163 \\
(100), 161(50) \\
119(60), 101 \\
(20)\end{array}$ & $91(100)$ & - & + & - & - & - & - \\
\hline 33 & 6.50 & $p$-Coumaric acid ${ }^{a}$ & $\mathrm{C}_{9} \mathrm{H}_{7} \mathrm{O}_{3}^{-}$ & 163.04007 & 163.03951 & 3.43 & $119(100)$ & $\begin{array}{c}119(60), 101 \\
(20), 93(25), \\
91(100), 72 \\
(10)\end{array}$ & - & + & + & - & + & + \\
\hline 34 & 6.58 & Gallic acid ethyl ester & $\mathrm{C}_{9} \mathrm{H}_{9} \mathrm{O}_{5}^{-}$ & 197.04555 & 197.04480 & 3.81 & $169(100)$ & $125(100)$ & $\begin{array}{c}107(10), 97 \\
(40), 81(100)\end{array}$ & + & + & - & - & - \\
\hline 35 & 6.75 & Ellagic acid $^{a}$ & $\mathrm{C}_{14} \mathrm{H}_{5} \mathrm{O}_{8}^{-}$ & 300.99899 & 300.99805 & 3.12 & $\begin{array}{c}284(40), 271 \\
(60), 257(100), \\
229(85), 185 \\
(40)\end{array}$ & $\begin{array}{l}229(100), 213 \\
(20), 185(85)\end{array}$ & $\begin{array}{c}201(100), 185 \\
(95), 157(30) \\
145(20), 129 \\
(10)\end{array}$ & + & + & - & - & + \\
\hline 36 & 6.76 & Sinapic acid ${ }^{a}$ & $\mathrm{C}_{11} \mathrm{H}_{11} \mathrm{O}_{5}^{-}$ & 223.06120 & 223.06047 & 3.27 & $\begin{array}{l}208(100), 179 \\
(30), 164(20)\end{array}$ & $\begin{array}{c}193(10), 164 \\
(100), 149(15) \\
135(5)\end{array}$ & $\begin{array}{c}149 \text { (100), } 135 \\
(35)\end{array}$ & - & + & - & - & - \\
\hline 37 & 7.05 & Ferulic acid $^{a}$ & $\mathrm{C}_{10} \mathrm{H}_{9} \mathrm{O}_{4}^{-}$ & 193.05063 & 193.04995 & 3.52 & $\begin{array}{c}178(70), 149 \\
(100), 134(50)\end{array}$ & $134(100)$ & $106(100)$ & + & + & - & - & + \\
\hline 38 & 7.67 & $\begin{array}{l}\text { Caffeoylcoumaroylquinic } \\
\text { acid }\end{array}$ & $\mathrm{C}_{25} \mathrm{H}_{23} \mathrm{O}_{11}^{-}$ & 499.12404 & 499.12375 & 0.58 & $\begin{array}{c}361(5), 337 \\
(100), 163(10)\end{array}$ & $\begin{array}{c}191(10), 173 \\
(60), 163(100) \\
119(10)\end{array}$ & $119(100)$ & - & + & - & - & - \\
\hline 39 & 8.01 & $\begin{array}{c}\text { Dihydroxybenzoic acid ethyl } \\
\text { ester }\end{array}$ & $\mathrm{C}_{9} \mathrm{H}_{9} \mathrm{O}_{4}^{-}$ & 181.05063 & 181.05011 & 2.87 & $\begin{array}{c}153(100), 109 \\
(10)\end{array}$ & $109(100)$ & - & + & - & - & + & - \\
\hline 40 & 8.02 & Caffeic acid methyl ester & $\mathrm{C}_{10} \mathrm{H}_{9} \mathrm{O}_{4}^{-}$ & 193.05063 & 193.05019 & 2.28 & $\begin{array}{c}178(30), 161 \\
(100), 134(70) \\
111(10)\end{array}$ & $133(100)$ & - & + & - & - & - & + \\
\hline 41 & 8.96 & Cinnamic acid $^{a}$ & $\mathrm{C}_{9} \mathrm{H}_{7} \mathrm{O}_{2}^{-}$ & 147.04515 & 147.04480 & 2.38 & $\begin{array}{l}104(10), 103 \\
(100), 87(10) \\
177(10), 162\end{array}$ & $119(100)$ & - & - & + & - & - & + \\
\hline 42 & 9.12 & $p$-Coumaric acid methyl ester & $\mathrm{C}_{10} \mathrm{H}_{9} \mathrm{O}_{3}^{-}$ & 177.05572 & 177.05504 & 3.84 & $\begin{array}{c}(40), 145(100) \\
118(50)\end{array}$ & $177(100)$ & - & + & - & - & + & + \\
\hline
\end{tabular}


Table 2. Cont.

\begin{tabular}{|c|c|c|c|c|c|c|c|c|c|c|c|c|c|c|}
\hline No & $t_{\mathrm{R}}, \min$ & Compound Name & $\begin{array}{c}\text { Molecular } \\
\text { Formula, } \\
{[\mathrm{M}-\mathrm{H}]^{-}}\end{array}$ & $\begin{array}{c}\text { Calculated } \\
\text { Mass, } \\
{[\mathrm{M}-\mathrm{H}]^{-}}\end{array}$ & $\begin{array}{r}\text { Exact } \\
\text { Mass, } \\
{[\mathrm{M}-\mathrm{H}]^{-}}\end{array}$ & $\Delta \mathrm{ppm}$ & $\begin{array}{c}\text { MS }^{2} \\
\text { Fragments, } \\
\text { (\% Base } \\
\text { Peak) }\end{array}$ & $\begin{array}{c}\text { MS }^{3} \\
\text { Fragments, } \\
\text { (\% Base } \\
\text { Peak) }\end{array}$ & $\begin{array}{c}\text { MS }^{4} \\
\text { Fragments, } \\
\text { (\% Base } \\
\text { Peak) }\end{array}$ & $\mathbf{W}$ & $\mathrm{S}$ & $\mathbf{P}$ & $\mathbf{E}$ & $\mathbf{L}$ \\
\hline \multicolumn{15}{|c|}{ Flavan-3-ols } \\
\hline 43 & 4.14 & $\begin{array}{l}\text { (Epi)catechin hexoside } \\
\text { isomer } 1\end{array}$ & $\mathrm{C}_{21} \mathrm{H}_{23} \mathrm{O}_{11}^{-}$ & 451.12404 & 451.12378 & 0.58 & $\begin{array}{c}289(100), 161 \\
(5)\end{array}$ & $\begin{array}{l}245(100), 205 \\
(40), 179(15)\end{array}$ & $\begin{array}{c}227(30), 203 \\
(100), 187(30), \\
175(10), 161 \\
(20)\end{array}$ & - & + & - & - & - \\
\hline 44 & 4.41 & Prodelphinidin dimer B type & $\mathrm{C}_{30} \mathrm{H}_{25} \mathrm{O}_{13}^{-}$ & 593.13006 & 593.12958 & 0.81 & $\begin{array}{c}467(15), 425 \\
(100), 407(30) \\
289(20)\end{array}$ & $\begin{array}{l}407(100), 381 \\
(5), 273(10)\end{array}$ & $\begin{array}{c}389(30), 297 \\
(30), 285(100) \\
243(70)\end{array}$ & + & + & - & - & - \\
\hline 45 & 4.70 & $\begin{array}{l}\text { Procyanidin dimer A type } \\
\text { isomer } 1\end{array}$ & $\mathrm{C}_{30} \mathrm{H}_{23} \mathrm{O}_{12}^{-}$ & 575.11950 & 575.11857 & 1.62 & $\begin{array}{c}449(80), 423 \\
(70), 407(100), \\
289(15), 287 \\
(35), 285(25)\end{array}$ & $\begin{array}{c}389(20), 297 \\
(30), 285(100) \\
243(30)\end{array}$ & $\begin{array}{c}257(100), 213 \\
(10)\end{array}$ & + & + & - & - & - \\
\hline 46 & 4.81 & $\begin{array}{l}\text { (Epi)catechin hexoside } \\
\text { isomer } 2\end{array}$ & $\mathrm{C}_{21} \mathrm{H}_{23} \mathrm{O}_{11}^{-}$ & 451.12404 & 451.12387 & 0.38 & $\begin{array}{c}289(100), 161 \\
(5)\end{array}$ & $\begin{array}{l}245(100), 205 \\
(40), 179(15)\end{array}$ & $\begin{array}{c}227(30), 203 \\
(100), 187(30), \\
175(10), 161 \\
(20)\end{array}$ & - & + & - & - & - \\
\hline 47 & 4.83 & Gallocatechin $^{a}$ & $\mathrm{C}_{15} \mathrm{H}_{13} \mathrm{O}_{7}^{-}$ & 305.06668 & 305.06592 & 2.49 & $\begin{array}{c}261(50), 221 \\
(70), 219(70) \\
179(100), 165 \\
(35)\end{array}$ & $\begin{array}{l}164(100), 151 \\
(40), 135(30)\end{array}$ & $\begin{array}{c}120(100), 108 \\
(20)\end{array}$ & + & + & - & - & - \\
\hline 48 & 4.85 & $\begin{array}{l}\text { Procyanidin dimer B type } \\
\text { isomer } 1\end{array}$ & $\mathrm{C}_{30} \mathrm{H}_{25} \mathrm{O}_{12}{ }^{-}$ & 577.13515 & 577.13391 & 2.15 & $\begin{array}{c}559(10), 451 \\
(30), 425(100) \\
407(50), 289 \\
(25), 287(10)\end{array}$ & $\begin{array}{l}407(100), 381 \\
(5), 273(10)\end{array}$ & $\begin{array}{c}389(30), 297 \\
(30), 285(100) \\
243(70)\end{array}$ & + & + & + & + & - \\
\hline 49 & 4.94 & $\begin{array}{l}\text { Procyanidin dimer A type } \\
\text { isomer } 2\end{array}$ & $\mathrm{C}_{30} \mathrm{H}_{23} \mathrm{O}_{12}{ }^{-}$ & 575.11950 & 575.11850 & 1.74 & $\begin{array}{c}449(70), 423 \\
(100), 407(60), \\
289(10), 287 \\
(20), 285(5)\end{array}$ & $\begin{array}{c}405(100), 313 \\
(35), 297(45) \\
285(25), 243 \\
(30)\end{array}$ & $\begin{array}{l}387(10), 283 \\
(10), 243(100)\end{array}$ & + & + & - & - & - \\
\hline 50 & 5.01 & $\begin{array}{l}\text { Procyanidin trimer B type } \\
\text { isomer } 1\end{array}$ & $\mathrm{C}_{45} \mathrm{H}_{37} \mathrm{O}_{18}^{-}$ & 865.19854 & 865.19684 & 1.96 & $\begin{array}{c}695(100), 577 \\
(60), 425(30) \\
407(30), 287 \\
(30)\end{array}$ & $\begin{array}{l}543(100), 451 \\
(45), 243(60)\end{array}$ & $\begin{array}{c}525(100), 391 \\
(40)\end{array}$ & + & + & - & - & - \\
\hline
\end{tabular}


Table 2. Cont

\begin{tabular}{|c|c|c|c|c|c|c|c|c|c|c|c|c|c|c|}
\hline No & $t_{\mathrm{R}}, \min$ & Compound Name & $\begin{array}{c}\text { Molecular } \\
\text { Formula, } \\
{[\mathrm{M}-\mathrm{H}]^{-}}\end{array}$ & $\begin{array}{c}\text { Calculated } \\
\text { Mass, } \\
{[\mathrm{M}-\mathrm{H}]^{-}}\end{array}$ & $\begin{array}{c}\text { Exact } \\
\text { Mass, } \\
{[\mathrm{M}-\mathrm{H}]^{-}}\end{array}$ & $\Delta \mathrm{ppm}$ & $\begin{array}{c}\text { MS }^{2} \\
\text { Fragments, } \\
\text { (\% Base } \\
\text { Peak) }\end{array}$ & $\begin{array}{c}\text { MS }^{3} \\
\text { Fragments, } \\
\text { (\% Base } \\
\text { Peak) }\end{array}$ & $\begin{array}{c}\text { MS }^{4} \\
\text { Fragments, } \\
\text { (\% Base } \\
\text { Peak) }\end{array}$ & $\mathbf{W}$ & $\mathrm{S}$ & $\mathbf{P}$ & $\mathbf{E}$ & L \\
\hline 51 & 5.07 & $\begin{array}{c}\text { Chalcan-flavan 3-ol dimer } \\
\text { isomer } 1\end{array}$ & $\mathrm{C}_{30} \mathrm{H}_{27} \mathrm{O}_{12}{ }^{-}$ & 579.15079 & 579.14984 & 1.64 & $\begin{array}{c}289 \text { (100), } 245 \\
(10)\end{array}$ & $\begin{array}{l}245(100), 205 \\
(30), 179(15)\end{array}$ & $\begin{array}{c}227(30), 203 \\
(100), 187(30) \\
175(10), 161 \\
(20)\end{array}$ & + & + & - & - & - \\
\hline 52 & 5.13 & $\begin{array}{l}\text { Procyanidin dimer B type } \\
\text { isomer } 2\end{array}$ & $\mathrm{C}_{30} \mathrm{H}_{25} \mathrm{O}_{12}^{-}$ & 577.13515 & 577.13403 & 1.94 & $\begin{array}{c}559(10), 451 \\
(30), 425(100) \\
407(50), 289 \\
(25), 287(10)\end{array}$ & $\begin{array}{l}407(100), 381 \\
(5), 273(10)\end{array}$ & $\begin{array}{c}389(30), 297 \\
(30), 285(100) \\
243(70)\end{array}$ & + & + & + & - & - \\
\hline 53 & 5.25 & $\begin{array}{l}\text { Procyanidin dimer A type } \\
\text { isomer } 3\end{array}$ & $\mathrm{C}_{30} \mathrm{H}_{23} \mathrm{O}_{12}^{-}$ & 575.11950 & 575.11830 & 2.09 & $\begin{array}{c}449(50), 423 \\
(100), 407(30), \\
289(5), 287 \\
(20), 285(10)\end{array}$ & $\begin{array}{c}405(100), 313 \\
(50), 297(30) \\
285(45), 243 \\
(40)\end{array}$ & $\begin{array}{c}387(20), 361 \\
(20), 243(100)\end{array}$ & + & + & - & - & - \\
\hline 54 & 5.26 & Catechin $^{a}$ & $\mathrm{C}_{15} \mathrm{H}_{13} \mathrm{O}_{6}{ }^{-}$ & 289.07176 & 289.07080 & 3.32 & $\begin{array}{c}271(5), 245 \\
(100), 205(40) \\
179(15), 125 \\
(5)\end{array}$ & $\begin{array}{c}227(30), 203 \\
(100), 187(25) \\
175(10), 161 \\
(20)\end{array}$ & $\begin{array}{c}188(70), 185 \\
(20), 175(100) \\
161(40), 157 \\
(10)\end{array}$ & + & + & + & + & + \\
\hline 55 & 5.31 & $\begin{array}{l}\text { Procyanidin dimer B type } \\
\text { isomer } 3\end{array}$ & $\mathrm{C}_{30} \mathrm{H}_{25} \mathrm{O}_{12}^{-}$ & 577.13515 & 577.13397 & 2.04 & $\begin{array}{c}425(25), 407 \\
(10), 329(10) \\
289(100), 287 \\
(80)\end{array}$ & $\begin{array}{l}245(100), 205 \\
(30), 179(15)\end{array}$ & $\begin{array}{c}227(30), 203 \\
(100), 187(30), \\
175(10), 161 \\
(20)\end{array}$ & + & + & + & - & - \\
\hline 56 & 5.36 & Epigallocatechin ${ }^{a}$ & $\mathrm{C}_{15} \mathrm{H}_{13} \mathrm{O}_{7}^{-}$ & 305.06668 & 305.06552 & 3.80 & $\begin{array}{c}261(40), 247 \\
(20), 221(90) \\
219(80), 179 \\
(100)\end{array}$ & $\begin{array}{l}164(100), 151 \\
(35), 135(30)\end{array}$ & $\begin{array}{c}120(100), 108 \\
(25)\end{array}$ & + & + & - & + & - \\
\hline 57 & 5.39 & $\begin{array}{l}\text { Procyanidin trimer B type } \\
\text { isomer } 2\end{array}$ & $\mathrm{C}_{45} \mathrm{H}_{37} \mathrm{O}_{18}{ }^{-}$ & 865.19854 & 865.19592 & 3.03 & $\begin{array}{c}695(100), 577 \\
(80), 425(30) \\
407(40), 287 \\
(35)\end{array}$ & $\begin{array}{l}543(100), 451 \\
(45), 243(60)\end{array}$ & $\begin{array}{c}525(100), 391 \\
(40)\end{array}$ & + & + & - & - & - \\
\hline 58 & 5.59 & $\begin{array}{c}\text { Chalcan-flavan 3-ol dimer } \\
\text { isomer } 2\end{array}$ & $\mathrm{C}_{30} \mathrm{H}_{27} \mathrm{O}_{12}^{-}$ & 579.15079 & 579.15002 & 1.33 & $\begin{array}{c}289(100), 245 \\
(10)\end{array}$ & $\begin{array}{l}245(100), 205 \\
(30), 179(15)\end{array}$ & $\begin{array}{c}227(30), 203 \\
(100), 187(30), \\
175(10), 161 \\
(20)\end{array}$ & + & + & - & - & - \\
\hline 59 & 5.64 & $\begin{array}{l}\text { Procyanidin trimer B type } \\
\text { isomer } 3\end{array}$ & $\mathrm{C}_{45} \mathrm{H}_{37} \mathrm{O}_{18}^{-}$ & 865.19854 & 865.19818 & 0.41 & $\begin{array}{c}695(100), 577 \\
(70), 425(30) \\
407(40), 287 \\
(30)\end{array}$ & $\begin{array}{l}543(100), 451 \\
(45), 243(60)\end{array}$ & $\begin{array}{c}525(100), 391 \\
(40)\end{array}$ & + & + & - & - & - \\
\hline
\end{tabular}


Table 2. Cont.

\begin{tabular}{|c|c|c|c|c|c|c|c|c|c|c|c|c|c|c|}
\hline No & $t_{\mathrm{R}}, \min$ & Compound Name & $\begin{array}{c}\text { Molecular } \\
\text { Formula, } \\
{[\mathrm{M}-\mathrm{H}]^{-}}\end{array}$ & $\begin{array}{c}\text { Calculated } \\
\text { Mass, } \\
{[\mathrm{M}-\mathrm{H}]^{-}}\end{array}$ & $\begin{array}{c}\text { Exact } \\
\text { Mass, } \\
{[\mathrm{M}-\mathrm{H}]^{-}}\end{array}$ & $\Delta \mathrm{ppm}$ & $\begin{array}{c}\text { MS }^{2} \\
\text { Fragments, } \\
\text { (\% Base } \\
\text { Peak) }\end{array}$ & $\begin{array}{c}\text { MS }^{3} \\
\text { Fragments, } \\
\text { (\% Base } \\
\text { Peak) }\end{array}$ & $\begin{array}{c}\text { MS }^{4} \\
\text { Fragments, } \\
\text { (\% Base } \\
\text { Peak) }\end{array}$ & $\mathbf{W}$ & $\mathrm{S}$ & $\mathbf{P}$ & $\mathbf{E}$ & $\mathbf{L}$ \\
\hline 60 & 5.91 & Epicatechin $^{a}$ & $\mathrm{C}_{15} \mathrm{H}_{13} \mathrm{O}_{6}{ }^{-}$ & 289.07176 & 289.07085 & 3.15 & $\begin{array}{c}271(5), 245 \\
(100), 205(40), \\
179(15), 125 \\
\text { (5) }\end{array}$ & $\begin{array}{c}227(35), 203 \\
(100), 187(30), \\
175(15), 161 \\
(25)\end{array}$ & $\begin{array}{c}188(60), 185 \\
(20), 175(100) \\
161(35), 157 \\
(15)\end{array}$ & + & + & + & + & + \\
\hline 61 & 6.20 & $\begin{array}{c}\text { Procyanidin trimer B type } \\
\text { isomer } 4\end{array}$ & $\mathrm{C}_{45} \mathrm{H}_{37} \mathrm{O}_{18}^{-}$ & 865.19854 & 865.19672 & 2.10 & $\begin{array}{c}695(100), 577 \\
(80), 425(35) \\
407(35), 287 \\
(30)\end{array}$ & $\begin{array}{l}543(100), 451 \\
(45), 243(60)\end{array}$ & $\begin{array}{c}525(100), 391 \\
(40)\end{array}$ & + & + & - & - & - \\
\hline 62 & 6.50 & Catechin 3-O-gallate $^{a}$ & $\mathrm{C}_{22} \mathrm{H}_{17} \mathrm{O}_{10}^{-}$ & 441.08272 & 441.08209 & 1.43 & $\begin{array}{c}331(10), 289 \\
(100), 271(10) \\
169(25)\end{array}$ & $\begin{array}{c}271(5), 245 \\
(100), 205(40) \\
179(20)\end{array}$ & $\begin{array}{c}227(20), 203 \\
(100), 187(20), \\
175(10), 161 \\
(20)\end{array}$ & - & + & - & - & - \\
\hline 63 & 6.68 & $\begin{array}{l}\text { (Epi)gallocatechin } \\
\text { 3-O-gallate methyl ether }\end{array}$ & $\mathrm{C}_{23} \mathrm{H}_{19} \mathrm{O}_{11}{ }^{-}$ & 471.09328 & 471.09219 & 2.31 & $\begin{array}{c}439(50), 287 \\
(100), 269(20), \\
169(20)\end{array}$ & $\begin{array}{c}243(15), 161 \\
(20), 125(100)\end{array}$ & $57(100)$ & - & + & + & - & - \\
\hline 64 & 7.68 & (Epi)catechin 3-O-vanillate & $\mathrm{C}_{23} \mathrm{H}_{19} \mathrm{O}_{9}^{-}$ & 439.10346 & 439.10275 & 1.62 & $\begin{array}{c}289(100), 287 \\
(90), 271(50) \\
269(30), 167 \\
(10)\end{array}$ & $\begin{array}{l}245(100), 205 \\
(30), 179(15)\end{array}$ & $\begin{array}{c}227(30), 203 \\
(100), 187(30) \\
175(10), 161 \\
(20)\end{array}$ & - & + & - & - & - \\
\hline 65 & 8.12 & (Epi)catechin 3-O-coumarate & $\mathrm{C}_{24} \mathrm{H}_{19} \mathrm{O}_{8}^{-}$ & 435.10854 & 435.10828 & 0.60 & $\begin{array}{c}341(15), 289 \\
(100), 269(25) \\
165(20)\end{array}$ & $\begin{array}{l}245 \text { (100), } 205 \\
(30), 179(15)\end{array}$ & $\begin{array}{c}227(30), 203 \\
(100), 187(30) \\
175(10), 161 \\
(20)\end{array}$ & - & + & - & - & - \\
\hline \multicolumn{15}{|c|}{ Flavanols } \\
\hline 66 & 6.09 & Myricetin 7-O-pentoside & $\mathrm{C}_{20} \mathrm{H}_{17} \mathrm{O}_{12}{ }^{-}$ & 449.07255 & 449.07162 & 2.07 & $317(100)$ & $\begin{array}{c}299(10), 289 \\
(60), 273(100), \\
255(40)\end{array}$ & $\begin{array}{c}255(100), 211 \\
(90)\end{array}$ & - & - & - & + & + \\
\hline 67 & 6.17 & Myricetin $^{a}$ & $\mathrm{C}_{15} \mathrm{H}_{9} \mathrm{O}_{8}{ }^{-}$ & 317.03029 & 317.02942 & 2.74 & $\begin{array}{c}299(10), 273 \\
(35), 207(100) \\
163(95)\end{array}$ & $\begin{array}{c}179 \text { (100), } 151 \\
(15)\end{array}$ & $151(100)$ & - & - & - & + & + \\
\hline 68 & 6.20 & Myricetin 3-O-hexoside & $\mathrm{C}_{21} \mathrm{H}_{19} \mathrm{O}_{13}{ }^{-}$ & 479.08311 & 479.08176 & 2.82 & $\begin{array}{c}317(90), 316 \\
(100)\end{array}$ & $\begin{array}{c}287(30), 271 \\
(100), 179(20) \\
151(10)\end{array}$ & $\begin{array}{c}271(10), 243 \\
(100), 227(30) \\
215(10), 199 \\
(10)\end{array}$ & + & - & - & + & + \\
\hline
\end{tabular}


Table 2. Cont.

\begin{tabular}{|c|c|c|c|c|c|c|c|c|c|c|c|c|c|c|}
\hline No & $t_{\mathrm{R}}, \min$ & Compound Name & $\begin{array}{c}\text { Molecular } \\
\text { Formula, } \\
{[\mathrm{M}-\mathrm{H}]^{-}}\end{array}$ & $\begin{array}{c}\text { Calculated } \\
\text { Mass, } \\
{[\mathrm{M}-\mathrm{H}]^{-}}\end{array}$ & $\begin{array}{l}\text { Exact } \\
\text { Mass, } \\
{[\mathrm{M}-\mathrm{H}]^{-}}\end{array}$ & $\Delta \mathrm{ppm}$ & $\begin{array}{c}\text { MS }^{2} \\
\text { Fragments, } \\
\text { (\% Base } \\
\text { Peak) }\end{array}$ & $\begin{array}{c}\text { MS }^{3} \\
\text { Fragments, } \\
\text { (\% Base } \\
\text { Peak) }\end{array}$ & $\begin{array}{c}\text { MS }^{4} \\
\text { Fragments, } \\
\text { (\% Base } \\
\text { Peak) }\end{array}$ & $\mathbf{W}$ & $\mathrm{S}$ & $\mathbf{P}$ & $\mathrm{E}$ & $\mathbf{L}$ \\
\hline 69 & 6.21 & $\begin{array}{l}\text { Quercetin } \\
\text { 3-O-hexuronide-7-O-hexoside }\end{array}$ & $\mathrm{C}_{27} \mathrm{H}_{27} \mathrm{O}_{18}^{-}$ & 639.12029 & 639.11902 & 1.99 & $\begin{array}{c}505(10), 477 \\
(100), 463(30) \\
301(50)\end{array}$ & $301(100)$ & $\begin{array}{c}273(20), 257 \\
(20), 179(100), \\
151(75)\end{array}$ & + & - & - & - & + \\
\hline 70 & 6.23 & $\begin{array}{c}\text { Quercetin } \\
\text { 3-O-(6"-rhamnosyl)hexoside }\end{array}$ & $\mathrm{C}_{27} \mathrm{H}_{29} \mathrm{O}_{16}{ }^{-}$ & 609.14611 & 609.14508 & 1.69 & $\begin{array}{c}343(5), 301 \\
(100), 300(30) \\
271(10), 255 \\
(5)\end{array}$ & $\begin{array}{c}273(25), 257 \\
(20), \mathbf{1 7 9}(100) \\
151(75)\end{array}$ & $151(100)$ & - & - & - & + & + \\
\hline 71 & 6.38 & $\begin{array}{l}\text { Myricetin } \\
\text { 3-O-hexuronide-7-O-hexoside }\end{array}$ & $\mathrm{C}_{27} \mathrm{H}_{27} \mathrm{O}_{19}{ }^{-}$ & 655.11520 & 655.11359 & 2.46 & $\begin{array}{c}521(20), 493 \\
(100), 479(30) \\
317(50)\end{array}$ & $317(100)$ & $\begin{array}{c}193(10), 179 \\
(100), 151(30)\end{array}$ & - & - & - & + & + \\
\hline 72 & 6.60 & Myricetin 7-O-hexuronide & $\mathrm{C}_{21} \mathrm{H}_{17} \mathrm{O}_{14}^{-}$ & 493.06238 & 493.06100 & 2.80 & $\begin{array}{c}359(10), 317 \\
(100)\end{array}$ & $\begin{array}{c}179(100), 151 \\
(40)\end{array}$ & $151(100)$ & - & - & - & + & + \\
\hline 73 & 6.67 & Quercetin 3-O-galactoside $^{a}$ & $\mathrm{C}_{21} \mathrm{H}_{19} \mathrm{O}_{12}{ }^{-}$ & 463.08820 & 463.08655 & 3.56 & $\begin{array}{c}301(100), 300 \\
(30)\end{array}$ & $\begin{array}{c}273(25), 257 \\
(20), \mathbf{1 7 9}(100) \\
151(75)\end{array}$ & $151(100)$ & + & + & + & + & + \\
\hline 74 & 6.81 & $\begin{array}{c}\text { Kaempferol } \\
\text { 7-O-(6"-rhamnosyl)hexoside }\end{array}$ & $\mathrm{C}_{27} \mathrm{H}_{29} \mathrm{O}_{15}^{-}$ & 593.15119 & 593.14905 & 3.61 & $285(100)$ & $\begin{array}{c}267(40), 257 \\
(100), 241(30), \\
229(40), 213 \\
(30)\end{array}$ & $\begin{array}{c}255(10), 239 \\
(30), 229(100) \\
163(40)\end{array}$ & - & - & - & - & + \\
\hline 75 & 6.88 & $\begin{array}{c}\text { Isorhamnetin } \\
\text { 3-O-(6"-rhamnosyl)hexoside }\end{array}$ & $\mathrm{C}_{28} \mathrm{H}_{31} \mathrm{O}_{16}{ }^{-}$ & 623.16176 & 623.15973 & 3.26 & $\begin{array}{c}315(100), 300 \\
(20), 271(10), \\
255(5)\end{array}$ & $\begin{array}{l}300(100), 287 \\
(5), 272(5)\end{array}$ & $\begin{array}{l}271(100), 255 \\
(50), 151(5)\end{array}$ & - & - & - & - & + \\
\hline 76 & 6.99 & Kaempferol 3-O-glucoside ${ }^{a}$ & $\mathrm{C}_{21} \mathrm{H}_{19} \mathrm{O}_{11}^{-}$ & 447.09329 & 447.09219 & 2.46 & $\begin{array}{c}327(20), 285 \\
(80), 284(100) \\
255(10)\end{array}$ & $\begin{array}{c}255(100), 227 \\
(10)\end{array}$ & $\begin{array}{c}227(100), 211 \\
(60)\end{array}$ & + & - & - & + & + \\
\hline 77 & 6.99 & Syringetin 3-O-hexoside & $\mathrm{C}_{23} \mathrm{H}_{23} \mathrm{O}_{13}{ }^{-}$ & 507.11441 & 507.11346 & 1.87 & $\begin{array}{c}479(10), 387 \\
(20), 345(80) \\
345(60), 344 \\
(100), 299(15)\end{array}$ & $\begin{array}{c}330(90), 316 \\
(100), 301(90), \\
287(10), 273 \\
(70)\end{array}$ & $\begin{array}{c}301(100), 300 \\
(20), 287(10) \\
273(60)\end{array}$ & + & - & - & + & - \\
\hline 78 & 7.01 & Isorhamnetin 3-O-hexoside & $\mathrm{C}_{22} \mathrm{H}_{21} \mathrm{O}_{12}{ }^{-}$ & 477.10385 & 477.10275 & 2.31 & $\begin{array}{l}357(20), 315 \\
(50), 314(100), \\
300(5), 299(5)\end{array}$ & $\begin{array}{c}300(30), 285 \\
(100), 271(75), \\
257(10), 243 \\
(25)\end{array}$ & $270(100)$ & - & + & + & + & - \\
\hline
\end{tabular}


Table 2. Cont.

\begin{tabular}{|c|c|c|c|c|c|c|c|c|c|c|c|c|c|c|}
\hline No & $t_{R}, \min$ & Compound Name & $\begin{array}{c}\text { Molecular } \\
\text { Formula, } \\
{[\mathrm{M}-\mathrm{H}]^{-}}\end{array}$ & $\begin{array}{c}\text { Calculated } \\
\text { Mass, } \\
{[\mathrm{M}-\mathrm{H}]^{-}}\end{array}$ & $\begin{array}{c}\text { Exact } \\
\text { Mass, } \\
{[\mathrm{M}-\mathrm{H}]^{-}}\end{array}$ & $\Delta$ ppm & $\begin{array}{c}\text { MS }^{2} \\
\text { Fragments, } \\
\text { (\% Base } \\
\text { Peak) }\end{array}$ & $\begin{array}{c}\text { MS }^{3} \\
\text { Fragments, } \\
\text { (\% Base } \\
\text { Peak) }\end{array}$ & $\begin{array}{c}\text { MS }^{4} \\
\text { Fragments, } \\
\text { (\% Base } \\
\text { Peak) }\end{array}$ & W & $\mathbf{S}$ & $\mathbf{P}$ & $\mathbf{E}$ & $\mathbf{L}$ \\
\hline 79 & 7.11 & Quercetin 3-O-hexuronide & $\mathrm{C}_{21} \mathrm{H}_{17} \mathrm{O}_{13}^{-}$ & 477.06692 & 477.06503 & 3.96 & $301(100)$ & $\begin{array}{c}273(20), 257 \\
(20), 179(100) \\
151(75)\end{array}$ & $151(100)$ & + & + & + & + & + \\
\hline 80 & 7.24 & $\begin{array}{l}\text { Quercetin 3-O-hexuronide } \\
\text { methyl ether }\end{array}$ & $\mathrm{C}_{22} \mathrm{H}_{19} \mathrm{O}_{13}^{-}$ & 491.08311 & 491.08102 & 4.26 & $\begin{array}{c}473(10), 315 \\
(50), 301(100) \\
300(60)\end{array}$ & $\begin{array}{c}283(15), 272 \\
(20), 256(10) \\
179(100), 151 \\
(75)\end{array}$ & $151(100)$ & - & - & - & + & + \\
\hline 81 & 7.36 & $\begin{array}{c}\text { Quercetin } \\
\text { 3-O-(6"-malonyl)hexoside }\end{array}$ & $\mathrm{C}_{24} \mathrm{H}_{21} \mathrm{O}_{15}{ }^{-}$ & 549.08859 & 549.08710 & 2.71 & $505(100)$ & $\begin{array}{c}463(30), 301 \\
(100), 300(50)\end{array}$ & $\begin{array}{c}273(15), 257 \\
(15), 179(100) \\
151(85)\end{array}$ & - & - & - & - & + \\
\hline 82 & 7.44 & Kaempferol 7-O-hexuronide & $\mathrm{C}_{21} \mathrm{H}_{17} \mathrm{O}_{12}^{-}$ & 461.07200 & 461.07101 & 2.15 & $285(100)$ & $\begin{array}{c}267(40), 257 \\
(100), 241(30), \\
229(50), 213 \\
(25)\end{array}$ & $\begin{array}{c}255(10), 239 \\
(30), 229(100) \\
163(60)\end{array}$ & + & - & - & + & + \\
\hline 83 & 7.73 & $\begin{array}{l}\text { Kaempferol 3-O-hexuronide } \\
\text { methyl ether }\end{array}$ & $\mathrm{C}_{22} \mathrm{H}_{19} \mathrm{O}_{12}^{-}$ & 475.08820 & 475.08667 & 3.22 & $\begin{array}{c}327(10), 301 \\
(10), 285(70) \\
284(100), 255 \\
(35)\end{array}$ & $\begin{array}{c}255(100), 227 \\
(10)\end{array}$ & $\begin{array}{c}227(100), 211 \\
(60)\end{array}$ & - & - & - & - & + \\
\hline 84 & 7.84 & $\begin{array}{c}\text { Kaempferol } \\
\text { 7-O-(6"-malonyl)hexoside }\end{array}$ & $\mathrm{C}_{24} \mathrm{H}_{21} \mathrm{O}_{14}^{-}$ & 533.09368 & 533.09204 & 3.08 & $489(100)$ & $285(100)$ & $\begin{array}{c}267(50), 257 \\
(100), 241(40) \\
229(60), 213 \\
(30)\end{array}$ & - & - & - & - & + \\
\hline 85 & 8.60 & Quercetin $^{a}$ & $\mathrm{C}_{15} \mathrm{H}_{9} \mathrm{O}_{7}^{-}$ & 301.03537 & 301.03391 & 4.85 & $\begin{array}{c}283(15), 271 \\
(60), 257(25) \\
179(100), 151 \\
(80)\end{array}$ & $151(100)$ & $\begin{array}{l}107(100), 83 \\
\quad(10)\end{array}$ & + & + & - & + & + \\
\hline 86 & 9.49 & Kaempferol $^{a}$ & $\mathrm{C}_{15} \mathrm{H}_{9} \mathrm{O}_{6}{ }^{-}$ & 285.04046 & 285.03958 & 3.09 & $\begin{array}{c}255(100), 227 \\
(10)\end{array}$ & $\begin{array}{l}211(100), 195 \\
(5), 167(15)\end{array}$ & $\begin{array}{l}211(40), 137 \\
\quad(100)\end{array}$ & + & - & - & - & + \\
\hline \multicolumn{15}{|c|}{ Stilbenes } \\
\hline 87 & 7.11 & Resveratrol hexoside & $\mathrm{C}_{20} \mathrm{H}_{21} \mathrm{O}_{8}^{-}$ & 389.12419 & 389.12230 & 4.86 & $227(100)$ & $\begin{array}{c}185(100), 183 \\
(40), 159(35) \\
157(30), 143 \\
(20)\end{array}$ & - & + & + & - & - & + \\
\hline
\end{tabular}


Table 2. Cont.

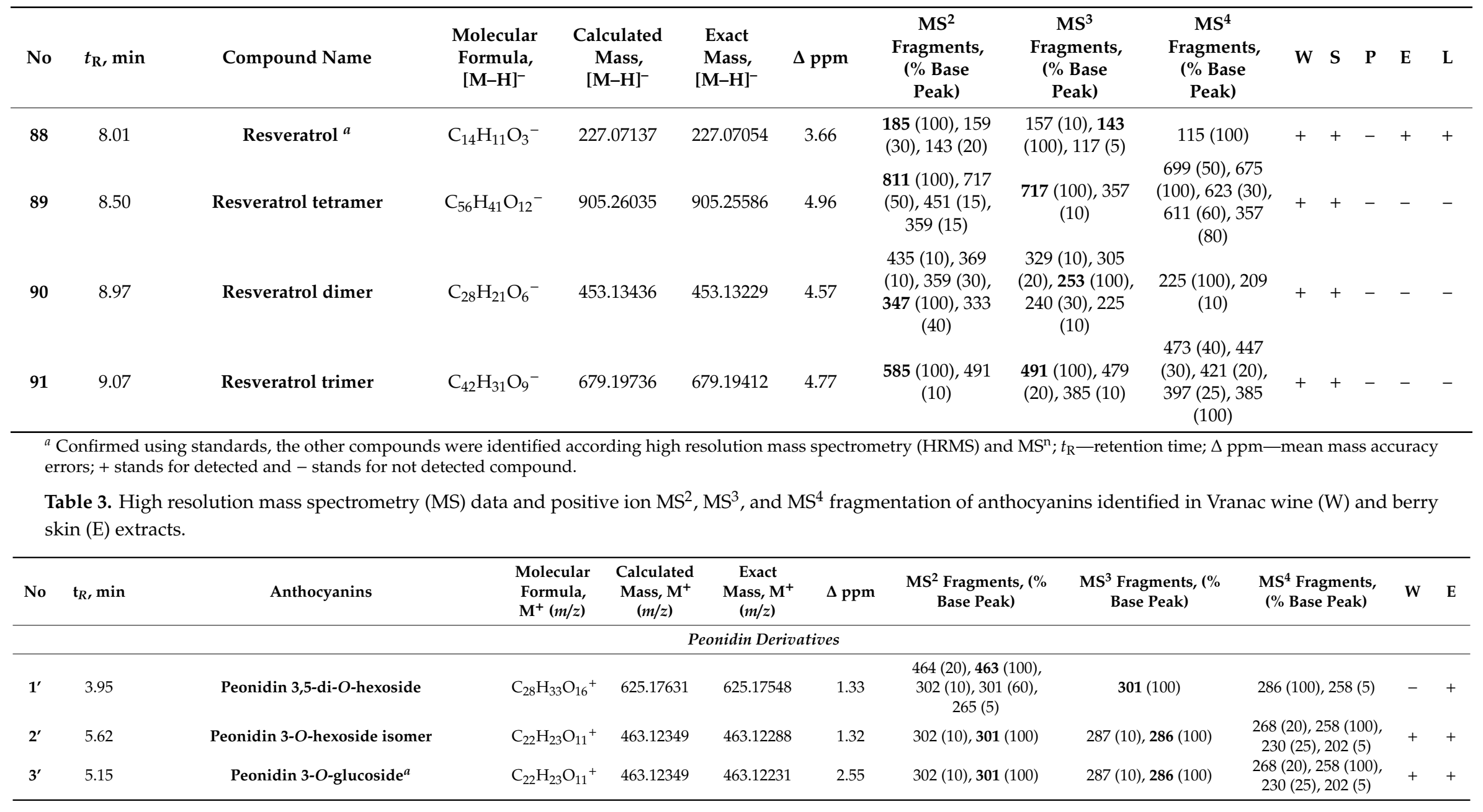


Table 3. Cont

\begin{tabular}{|c|c|c|c|c|c|c|c|c|c|c|c|}
\hline No & $\mathbf{t}_{R}, \min$ & Anthocyanins & $\begin{array}{c}\text { Molecular } \\
\text { Formula, } \\
\mathbf{M}^{+}(m / z)\end{array}$ & $\begin{array}{l}\text { Calculated } \\
\text { Mass, } \mathbf{M}^{+} \\
(\mathrm{m} / z)\end{array}$ & $\begin{array}{c}\text { Exact } \\
\text { Mass, } \mathbf{M}^{+} \\
(\mathrm{m} / \mathrm{z})\end{array}$ & $\Delta$ ppm & $\begin{array}{l}\text { MS }^{2} \text { Fragments, }(\% \\
\text { Base Peak) }\end{array}$ & $\begin{array}{l}\text { MS }^{3} \text { Fragments, }(\% \\
\text { Base Peak) }\end{array}$ & $\begin{array}{l}\text { MS }{ }^{4} \text { Fragments, } \\
\text { (\% Base Peak) }\end{array}$ & $\mathbf{W}$ & $\mathrm{E}$ \\
\hline $4^{\prime}$ & 5.70 & Peonidin 3-O-hexoside-pyruvate & $\mathrm{C}_{25} \mathrm{H}_{25} \mathrm{O}_{13}{ }^{+}$ & 531.11332 & 531.11255 & 1.45 & $370(15), 369(100)$ & $354(100), 326(10)$ & $\begin{array}{l}336(25), 326(100) \\
298(30), 253(30)\end{array}$ & + & + \\
\hline $5^{\prime}$ & 6.08 & Peonidin 3-O-(6"-acetyl)hexoside & $\mathrm{C}_{24} \mathrm{H}_{25} \mathrm{O}_{12}{ }^{+}$ & 505.13405 & 505.13358 & 0.93 & $302(10), 301(100)$ & $287(10), 286(100)$ & $\begin{array}{c}268(20), 258(100), \\
230(25), 202(5)\end{array}$ & + & + \\
\hline $6^{\prime}$ & 6.48 & Peonidin 3-O-(6"-caffeloyl)hexoside & $\mathrm{C}_{31} \mathrm{H}_{29} \mathrm{O}_{14}{ }^{+}$ & 625.15518 & 625.15369 & 2.38 & $\begin{array}{l}317(10), 302(15), 301 \\
\quad(100), 286(10)\end{array}$ & $287(5), 286(100)$ & $\begin{array}{c}268(20), 258(100), \\
257(20), 230(30), \\
202(10)\end{array}$ & - & + \\
\hline $7^{\prime}$ & 6.80 & Peonidin 3-O-(6"-p-coumaroyl)hexoside & $\mathrm{C}_{31} \mathrm{H}_{29} \mathrm{O}_{13}{ }^{+}$ & 609.16027 & 609.15796 & 3.79 & $302(10), 301(100)$ & $287(10), 286(100)$ & $\begin{array}{l}268(20), 258(100), \\
230(25), 202(5)\end{array}$ & + & + \\
\hline \multicolumn{12}{|c|}{ Delphinidin Derivatives } \\
\hline $8^{\prime}$ & 4.50 & Delphinidin 3-O-glucoside ${ }^{a}$ & $\mathrm{C}_{21} \mathrm{H}_{21} \mathrm{O}_{12}{ }^{+}$ & 465.10275 & 465.10123 & 3.27 & $304(15), 303(100)$ & $\begin{array}{l}285(10), 275(10), 257 \\
(100), 247(15), 229(30)\end{array}$ & $\begin{array}{c}239(5), 229(100), \\
213(25), 201(25), \\
173(20)\end{array}$ & + & + \\
\hline $9^{\prime}$ & 4.85 & Delphinidin 3-O-hexoside-pyruvate & $\mathrm{C}_{24} \mathrm{H}_{21} \mathrm{O}_{14}^{+}$ & 533.09258 & 533.09113 & 2.72 & $372(10), 371(100)$ & $\begin{array}{c}371(30), 353(10), 343 \\
(10), 325(100), 315(10)\end{array}$ & $297(100), 281(80)$ & + & + \\
\hline $10^{\prime}$ & 5.45 & Delphinidin 3-O-(6"-acetyl)hexoside & $\mathrm{C}_{23} \mathrm{H}_{23} \mathrm{O}_{13}{ }^{+}$ & 507.11332 & 507.11270 & 1.22 & $304(10), 303(100)$ & $\begin{array}{c}303(20), 285(10), 257 \\
(100), 247(10), 229(30)\end{array}$ & $\begin{array}{c}229(100), 213(25), \\
201(30), 173(15)\end{array}$ & + & + \\
\hline $11^{\prime}$ & 6.22 & Delphinidin 3-O-(6"-p-coumaroyl)hexoside & $\mathrm{C}_{30} \mathrm{H}_{27} \mathrm{O}_{14}^{+}$ & 611.13953 & 611.13904 & 0.80 & $304(10), 303(100)$ & $\begin{array}{l}303(20), 285(10), 257 \\
(100), 247(10), 229(30)\end{array}$ & $\begin{array}{l}229(100), 213(25) \\
201(30), 173(15)\end{array}$ & + & + \\
\hline $12^{\prime}$ & 6.53 & Delphinidin 3-O-hexoside isomer & $\mathrm{C}_{21} \mathrm{H}_{21} \mathrm{O}_{12}{ }^{+}$ & 465.10275 & 465.10202 & 1.57 & $304(15), 303(100)$ & $\begin{array}{l}285(60), 275(10), 257 \\
(100), 247(30), 229(80)\end{array}$ & $\begin{array}{c}239(5), 229(100) \\
213(5), 201(10) \\
173(5)\end{array}$ & + & + \\
\hline $13^{\prime}$ & 6.56 & Delphinidin 3-O-hexuronide & $\mathrm{C}_{21} \mathrm{H}_{19} \mathrm{O}_{13}{ }^{+}$ & 479.08202 & 479.08124 & 1.63 & $304(10), 303(100)$ & $\begin{array}{c}285(50), 274(20), 257 \\
(100), 247(30), 229(80)\end{array}$ & 229 (100), $201(10)$ & + & + \\
\hline \multicolumn{12}{|c|}{ Malvidin Derivatives } \\
\hline $14^{\prime}$ & 4.62 & Malvidin 3,5-di-O-glucoside ${ }^{a}$ & $\mathrm{C}_{29} \mathrm{H}_{35} \mathrm{O}_{17}+$ & 655.18688 & 655.18469 & 3.34 & $\begin{array}{l}494(15), 493(100), \\
332(10), 331(70)\end{array}$ & $331(100)$ & $\begin{array}{c}316(80), 315(100), \\
299(90), 298(30), \\
287(70)\end{array}$ & + & + \\
\hline $15^{\prime}$ & 5.35 & Malvidin 3-O-glucoside $^{a}$ & $\mathrm{C}_{23} \mathrm{H}_{25} \mathrm{O}_{12}{ }^{+}$ & 493.13405 & 493.13300 & 2.13 & $332(10), 331(100)$ & $\begin{array}{l}316(80), 315(100), 299 \\
(90), 298(30), 287(70)\end{array}$ & $\begin{array}{c}313(90), 299(25), \\
287(100), 285(70), \\
257(60)\end{array}$ & + & + \\
\hline $16^{\prime}$ & 5.66 & $\begin{array}{l}\text { Malvidin 3-O-hexoside-acetaldehyde(Vitisin } \\
\text { B) }\end{array}$ & $\mathrm{C}_{25} \mathrm{H}_{25} \mathrm{O}_{12}{ }^{+}$ & 517.13405 & 517.13336 & 1.33 & $356(15), 355(100)$ & $\begin{array}{l}339(100), 322(40), 311 \\
(15), 294(45), 266(20)\end{array}$ & $\begin{array}{c}337(50), 321(35), \\
311(100), 309(95), \\
293(10)\end{array}$ & + & + \\
\hline
\end{tabular}


Table 3. Cont.

\begin{tabular}{|c|c|c|c|c|c|c|c|c|c|c|c|}
\hline No & $\mathbf{t}_{R}, \min$ & Anthocyanins & $\begin{array}{c}\text { Molecular } \\
\text { Formula, } \\
\mathbf{M}^{+}(m / z)\end{array}$ & $\begin{array}{c}\text { Calculated } \\
\text { Mass, } \mathbf{M}^{+} \\
(\mathrm{m} / \mathrm{z})\end{array}$ & $\begin{array}{c}\text { Exact } \\
\text { Mass, } \mathbf{M}^{+} \\
(\mathrm{m} / \mathrm{z})\end{array}$ & $\Delta \mathrm{ppm}$ & $\begin{array}{l}\text { MS }^{2} \text { Fragments, }(\% \\
\text { Base Peak) }\end{array}$ & $\begin{array}{l}\text { MS }^{3} \text { Fragments, }(\% \\
\text { Base Peak) }\end{array}$ & $\begin{array}{l}\text { MS }{ }^{4} \text { Fragments, } \\
\text { (\% Base Peak) }\end{array}$ & $\mathbf{W}$ & $\mathrm{E}$ \\
\hline $17^{\prime}$ & 5.69 & Malvidin 3-O-pentoside & $\mathrm{C}_{22} \mathrm{H}_{23} \mathrm{O}_{11}{ }^{+}$ & 463.12349 & 463.12347 & 0.04 & $\begin{array}{c}332(5), 331(100), 301 \\
(10)\end{array}$ & $\begin{array}{l}316(90), 315(100), 299 \\
(85), 298(30), 287(60)\end{array}$ & $\begin{array}{c}313(90), 299(25) \\
287(100), 285(70) \\
257(60)\end{array}$ & + & + \\
\hline $18^{\prime}$ & 5.77 & Malvidin 3-O-hexoside-pyruvate(Vitisin A) & $\mathrm{C}_{26} \mathrm{H}_{25} \mathrm{O}_{14}{ }^{+}$ & 561.12388 & 561.12244 & 2.57 & $400(10), 399(100)$ & $\begin{array}{l}383(100), 367(70), 355 \\
(30), 338(80), 310(40)\end{array}$ & $\begin{array}{c}381(60), 365(40) \\
355(100), 337(15), \\
325(20)\end{array}$ & + & + \\
\hline $19^{\prime}$ & 5.91 & Malvidin 3-O-hexoside-acetone & $\mathrm{C}_{26} \mathrm{H}_{27} \mathrm{O}_{12}{ }^{+}$ & 531.14970 & 531.14911 & 1.11 & $\begin{array}{l}370(10), 369(100) \\
325(5)\end{array}$ & $\begin{array}{l}353(100), 336(20), 325 \\
(10), 308(25), 280(15)\end{array}$ & $\begin{array}{c}351(60), 335(30) \\
325(100), 323(80), \\
295(25)\end{array}$ & + & + \\
\hline $20^{\prime}$ & 6.03 & Malvidin 3-O-(6"-acetyl)hexoside-pyruvate & $\mathrm{C}_{28} \mathrm{H}_{27} \mathrm{O}_{15}{ }^{+}$ & 603.13445 & 603.13416 & 0.48 & $400(15), 399(100)$ & $\begin{array}{l}383(100), 367(80), 355 \\
(40), 338(80), 310(40)\end{array}$ & $\begin{array}{c}381(60), 365(40) \\
355(100), 337(20), \\
325(20)\end{array}$ & + & - \\
\hline $21^{\prime}$ & 6.13 & Malvidin 3-O-(6"-acetyl)hexoside & $\mathrm{C}_{25} \mathrm{H}_{27} \mathrm{O}_{13}{ }^{+}$ & 535.14462 & 535.14331 & 2.45 & $332(10), 331(100)$ & $\begin{array}{l}316(80), 315(100), 299 \\
(90), 298(30), 287(70)\end{array}$ & $\begin{array}{c}313(100), 299(30) \\
287(90), 285(80) \\
257(60)\end{array}$ & + & + \\
\hline $22^{\prime}$ & 6.39 & Malvidin 3-O-(6"-caffeoyl)hexoside & $\mathrm{C}_{32} \mathrm{H}_{31} \mathrm{O}_{15}{ }^{+}$ & 655.16575 & 655.16406 & 2.58 & $332(10), 331(100)$ & $\begin{array}{l}316(80), 315(100), 299 \\
(90), 298(30), 287(70)\end{array}$ & $\begin{array}{c}313(90), 299(25) \\
287(100), 285(70) \\
257(60)\end{array}$ & - & + \\
\hline $23^{\prime}$ & 6.70 & $\begin{array}{c}\text { Malvidin } \\
\text { 3-O-(6"-p-coumaroyl)hexoside-pyruvate }\end{array}$ & $\mathrm{C}_{35} \mathrm{H}_{31} \mathrm{O}_{16}{ }^{+}$ & 707.16066 & 707.15979 & 1.23 & $400(10), 399(100)$ & $\begin{array}{l}383(100), 367(70), 355 \\
(30), 338(80), 310(40)\end{array}$ & $\begin{array}{c}381(60), 365(40) \\
355(100), 337(15), \\
325(20)\end{array}$ & + & + \\
\hline $24^{\prime}$ & 6.76 & $\begin{array}{l}\text { Malvidin 3-O-hexoside-4-vinylcatechol } \\
\text { (Pinotin A) }\end{array}$ & $\mathrm{C}_{31} \mathrm{H}_{29} \mathrm{O}_{14}^{+}$ & 625.15518 & 625.15440 & 1.25 & $464(15), 463(100)$ & $\begin{array}{l}448(50), 447(100), 419 \\
(10), 402(20), 374(20)\end{array}$ & $\begin{array}{c}447(50), 445(65) \\
429(20), 419(100) \\
417(95)\end{array}$ & + & - \\
\hline $25^{\prime}$ & 6.84 & Malvidin 3-O-(6"-p-coumaroyl)hexoside & $\mathrm{C}_{32} \mathrm{H}_{31} \mathrm{O}_{14}{ }^{+}$ & 639.17083 & 639.16996 & 1.36 & $332(10), 331(100)$ & $\begin{array}{l}316(80), 315(100), 299 \\
(90), 298(30), 287(70)\end{array}$ & $\begin{array}{c}313(90), 299(25) \\
287(100), 285(70) \\
257(60)\end{array}$ & + & + \\
\hline $26^{\prime}$ & 6.89 & Malvidin 3-O-(6"-feruloyl)hexoside & $\mathrm{C}_{33} \mathrm{H}_{33} \mathrm{O}_{15}{ }^{+}$ & 669.18140 & 669.17857 & 4.23 & $332(10), 331(100)$ & $\begin{array}{l}316(80), 315(100), 299 \\
(90), 298(30), 287(70)\end{array}$ & $\begin{array}{c}313(90), 299(25) \\
287(100), 285(70) \\
257(60)\end{array}$ & - & + \\
\hline $27^{\prime}$ & 6.99 & Malvidin 3-O-hexoside-4-vinylphenol & $\mathrm{C}_{31} \mathrm{H}_{29} \mathrm{O}_{13}{ }^{+}$ & 609.16027 & 609.16022 & 0.08 & $448(25), 447(100)$ & $\begin{array}{l}432(50), 431(100), 414 \\
(15), 386(20), 358(20)\end{array}$ & $\begin{array}{c}431(50), 429(65) \\
413(20), 403(100) \\
401(95)\end{array}$ & + & - \\
\hline $28^{\prime}$ & 7.19 & Malvidin 3-O-hexoside-pyranone & $\mathrm{C}_{25} \mathrm{H}_{25} \mathrm{O}_{13}{ }^{+}$ & 533.12897 & 533.12677 & 4.13 & $372(10), 371(100)$ & $\begin{array}{l}356(15), 343(100), 339 \\
(10), 311(30), 283(20)\end{array}$ & $\begin{array}{l}311(100), 283(60) \\
265(5), 255(15)\end{array}$ & + & - \\
\hline
\end{tabular}


Table 3. Cont

\begin{tabular}{|c|c|c|c|c|c|c|c|c|c|c|c|}
\hline No & $\mathbf{t}_{R}, \min$ & Anthocyanins & $\begin{array}{c}\text { Molecular } \\
\text { Formula, } \\
\mathbf{M}^{+}(m / z)\end{array}$ & $\begin{array}{l}\text { Calculated } \\
\text { Mass, } \mathbf{M}^{+} \\
(\mathrm{m} / z)\end{array}$ & $\begin{array}{c}\text { Exact } \\
\text { Mass, } \mathbf{M}^{+} \\
(\mathrm{m} / \mathrm{z})\end{array}$ & $\Delta \mathrm{ppm}$ & $\begin{array}{l}\text { MS }^{2} \text { Fragments, ( } \% \\
\text { Base Peak) }\end{array}$ & $\begin{array}{l}\text { MS }^{3} \text { Fragments, }(\% \\
\text { Base Peak) }\end{array}$ & $\begin{array}{l}\text { MS }{ }^{4} \text { Fragments, } \\
\text { (\% Base Peak) }\end{array}$ & $\mathbf{W}$ & $E$ \\
\hline $29^{\prime}$ & 7.30 & $\begin{array}{c}\text { Malvidin } \\
\text { 3-O-(6"-acetyl)hexoside-4-vinylphenol }\end{array}$ & $\mathrm{C}_{33} \mathrm{H}_{31} \mathrm{O}_{14}{ }^{+}$ & 651.17083 & 651.17035 & 0.74 & $448(25), 447(100)$ & $\begin{array}{l}432(50), 431(100), 414 \\
(15), 403(10), 386(20)\end{array}$ & $\begin{array}{c}431(50), 429(65), \\
413(20), 403(100), \\
401(95)\end{array}$ & + & - \\
\hline $30^{\prime}$ & 7.40 & Malvidin-pyruvate & $\mathrm{C}_{20} \mathrm{H}_{15} \mathrm{O}_{9}^{+}$ & 399.07106 & 399.07031 & 1.88 & $382(20), 381(100)$ & $\begin{array}{l}366(10), 353(100), 337 \\
(10), 325(90), 297(60)\end{array}$ & $\begin{array}{l}338(5), 325(100) \\
309(10), 297(25)\end{array}$ & + & - \\
\hline $31^{\prime}$ & 7.55 & $\begin{array}{c}\text { Malvidin } \\
\text { 3-O-(6"-p-coumaroyl)hexoside-4-vinylphenol }\end{array}$ & $\mathrm{C}_{40} \mathrm{H}_{35} \mathrm{O}_{15}{ }^{+}$ & 755.19705 & 755.19592 & 1.50 & $448(25), 447(100)$ & $\begin{array}{l}432(50), 431(100), 414 \\
(15), 403(10), 386(20)\end{array}$ & $\begin{array}{c}431(50), 429(65), \\
413(20), 403(100), \\
401(95)\end{array}$ & + & - \\
\hline $32^{\prime}$ & 7.30 & $\begin{array}{c}\text { Malvidin } \\
\text { 3-O-(6"-p-coumaroyl)hexoside-4-vinylcatechol }\end{array}$ & $\mathrm{C}_{40} \mathrm{H}_{35} \mathrm{O}_{16}+$ & 771.19196 & 771.19055 & 1.83 & $464(15), 463(100)$ & $\begin{array}{l}448(50), 447(100), 430 \\
(15), 419(10), 402(20)\end{array}$ & $\begin{array}{c}447(50), 445(65) \\
429(20), 419(100), \\
417(95)\end{array}$ & + & - \\
\hline \multicolumn{12}{|c|}{ Cyanidin Derivatives } \\
\hline $33^{\prime}$ & 4.78 & Cyanidin 3-O-glucoside ${ }^{a}$ & $\mathrm{C}_{21} \mathrm{H}_{21} \mathrm{O}_{11}{ }^{+}$ & 449.10784 & 449.10684 & 2.23 & $288(10), 287(100)$ & $\begin{array}{l}269(25), 259(40), 241 \\
(50), 231(70), 213(100)\end{array}$ & $\begin{array}{c}195(10), 185(100), \\
167(10), 157(20), \\
141(20)\end{array}$ & + & + \\
\hline $34^{\prime}$ & 5.04 & Cyanidin 3-O-arabinoside ${ }^{a}$ & $\mathrm{C}_{20} \mathrm{H}_{19} \mathrm{O}_{10}{ }^{+}$ & 419.09727 & 419.09613 & 2.72 & $288(10), 287(100)$ & $\begin{array}{l}269(25), 259(40), 241 \\
(50), 231(70), 213(100)\end{array}$ & $\begin{array}{c}195(10), 185(100), \\
167(10), 157(20) \\
141(20)\end{array}$ & + & + \\
\hline $35^{\prime}$ & 6.43 & Cyanidin 3-O-(6"-p-coumaroyl)hexoside & $\mathrm{C}_{30} \mathrm{H}_{27} \mathrm{O}_{13}^{+}$ & 595.14462 & 595.14374 & 1.48 & $288(10), 287(100)$ & $\begin{array}{l}269(25), 259(40), 241 \\
(50), 231(70), 213(100)\end{array}$ & $\begin{array}{c}195(10), 185(100), \\
167(10), 157(20) \\
141(20)\end{array}$ & - & + \\
\hline \multicolumn{12}{|c|}{ Petunidin Derivatives } \\
\hline $36^{\prime}$ & 4.89 & Petunidin 3-O-hexoside & $\mathrm{C}_{22} \mathrm{H}_{23} \mathrm{O}_{12}+$ & 479.11840 & 479.11655 & 3.86 & $318(10), 317(100)$ & 302 (100), 274 (5) & $\begin{array}{l}274(100), 246(10), \\
228(10), 218(10)\end{array}$ & + & + \\
\hline $37^{\prime}$ & 5.79 & Petunidin 3-O-(6"-acetyl)hexoside & $\mathrm{C}_{24} \mathrm{H}_{25} \mathrm{O}_{13}{ }^{+}$ & 521.12897 & 521.12872 & 0.48 & $318(10), 317(100)$ & $\begin{array}{c}302(100), 285(30), 274 \\
(10)\end{array}$ & $\begin{array}{l}285(10), 274(100) \\
246(15), 153(20)\end{array}$ & + & + \\
\hline \multicolumn{12}{|c|}{ Pelargonidin Derivatives } \\
\hline $38^{\prime}$ & 5.17 & Pelargonidin 3-O-(6"-rhamnosyl)glucoside ${ }^{a}$ & $\mathrm{C}_{27} \mathrm{H}_{31} \mathrm{O}_{14}^{+}$ & 579.17083 & 579.16989 & 1.62 & $\begin{array}{l}434(5), 433(15), 272 \\
(10), 271(100)\end{array}$ & $\begin{array}{l}271(100), 253(40), 225 \\
(60), 215(70), 197(80)\end{array}$ & $\begin{array}{l}197(5), 169(100), \\
153(5), 141(30)\end{array}$ & + & + \\
\hline
\end{tabular}

${ }^{a}$ Confirmed using standards, the other compounds were identified according to high resolution mass spectrometry (HRMS) and MS ${ }^{\mathrm{n}} t_{\mathrm{R}}-\mathrm{retention}$ time; $\Delta$ ppm-mean mass accuracy errors; + stands for detected and - stands for not detected compound. 
A

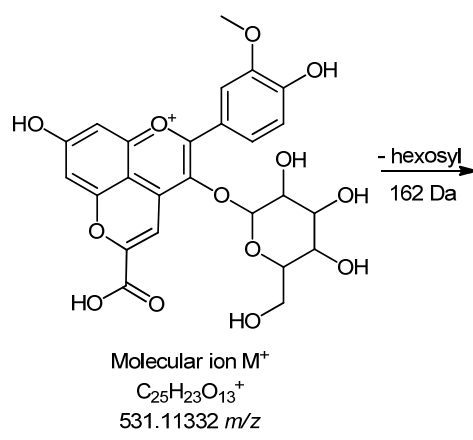
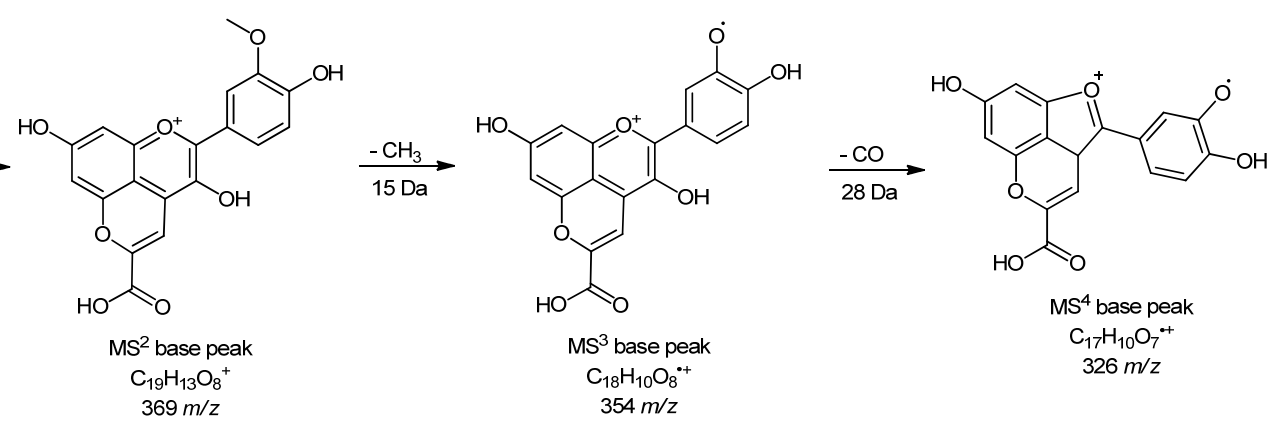

B

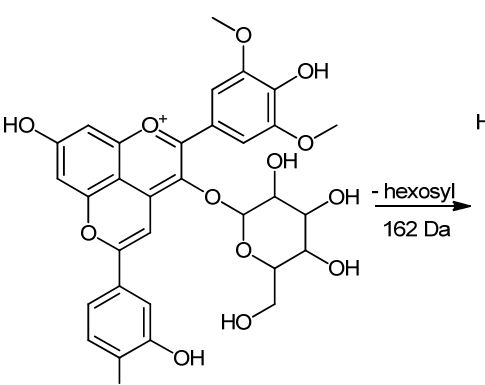

$\mathrm{OH}$

Molecular ion $\mathrm{M}^{+}$

$\mathrm{C}_{31} \mathrm{H}_{29} \mathrm{O}_{14}$

$625.15518 \mathrm{~m}$
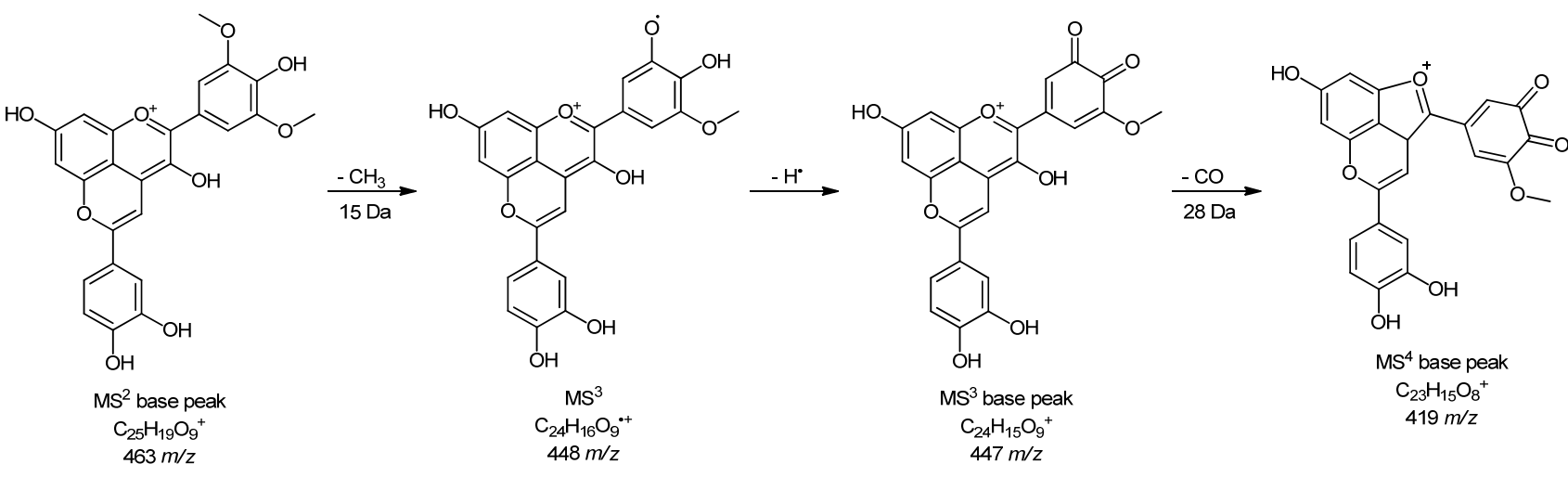

Figure 2. Fragmentation pathway of (A) peonidin 3-O-hexoside-pyruvate (4') and (B) malvidin 3-O-hexoside-4-vinylcatechol (24') in positive ionization mode. 
Peonidin 3-O-hexoside-pyruvate was found in both investigated samples (wine and grape skin) at $5.70 \mathrm{~min}$ and molecular ion at $531 \mathrm{~m} / \mathrm{z}$. It gave a MS² base peak at $369 \mathrm{~m} / \mathrm{z}$ generated by loss of hexosyl group (162 Da). Further loss of methyl group (15 Da) gave a MS ${ }^{3}$ base peak at $354 \mathrm{~m} / \mathrm{z}$. MS ${ }^{4}$ base peak was formed by elimination of $\mathrm{CO}(28 \mathrm{Da})$ from A ring of anthocyanin.

In addition, a significant number of pyranoanthocyanins were found, which are known to be made by maturation and aging of wine [37]. These compounds represent stable pigments that are mostly formed during fermentation of must by reaction between free anthocyanins and certain yeast by-products (acetaldehyde, pyruvic acid and vinylphenols [38]). In our study, young wine was examined so that a large number of these compounds were not found.

Malvidin 3-O-hexoside-pyruvate (18') or vitisin A and malvidin 3-O-hexoside-4-vinylphenol (27') were earlier identified in Vranac wine [39]. Malvidin 3-O-hexoside-4-vinylcatechol or pinotin A (24') was found only in wine sample at $6.76 \mathrm{~min}$ and $625 \mathrm{~m} / \mathrm{z}$. The detailed fragmentation pathway of this compound is depicted in Figure 2B.

\section{Conclusions}

In order to study in detail the polyphenolic compounds of the autochthonous grapevine variety Vranac (Podgorica region, Montenegro), the leaf, skin, seed, pulp and young Vranac wine were analyzed. To the best of our knowledge, for the first time total phenolic content and radical scavenging activity were determined in individual parts of grape berry and leaves. As expected, the highest TPC and TAC were found in seeds, followed by leaves, while the lowest values were obtained for skin and pulp.

LC-MS analyses revealed a total of 91 polyphenolic compounds (phenolic acid derivatives, flavan-3-ols, flavanols, and stilbenes) in the negative ionization mode, while in positive ionization mode 38 anthocyanins were identified. Using high-resolution mass spectrometry (HRMS) in combination with $\mathrm{MS}^{\mathrm{n}}$ fragmentation, it was observed that chlorogenic acid and ellagic acid pentoside were found only in the Vranac leaf sample. The largest number of compounds from the group of flavan-3-ols (monomers, dimers, and trimers) has been identified in wine and grape seed samples. The largest number of anthocyanins, found in Vranac wine and berry skin extracts, were derivatives of malvidin.

Based on the obtained results, it can be concluded that the examined grapevine variety Vranac from Podgorica region has a wide range of diverse phenolic compounds. In addition, the results obtained in this work could give important data on the distribution of polyphenols throughout the plant (leaves and grape berries), along with wine. This information can assist in the selection of technology for the production of wines with a high amount of active ingredients.

Finally, as far as we know, tentative identification of (epi)catechin 3-O-coumarate in Vranac grape seed and chalcan-flavan 3-ol dimers in Vranac wine and grape seed is reported for the first time in this study. These types of compounds have not been found in any grapevine variety so far.

Supplementary Materials: The following are available online at http://www.mdpi.com/2304-8158/9/2/138/s1, Figure S1: sketch of all three vineyards.

Author Contributions: Conceptualization, D.Š. and Ž.T.; methodology, S.T.; software, U.G.; validation, U.G., M.S. and I.Ć.; formal analysis, D.Š.; investigation, D.Š.; resources, B.K.; data curation, J.M.; writing一original draft preparation, U.G.; writing_-review and editing, J.M.; funding acquisition, Ž.T. All authors have read and agreed to the published version of the manuscript.

Funding: This work was supported by the Ministry of Education, Science, and Technological Development of Serbia, Grant Nos. 172017 and 173024.

Conflicts of Interest: The authors declare no conflict of interest. 


\section{References}

1. Jordão, A.M.; Simões, S.; Correia, A.C.; Gonçalves, F.J. Antioxidant activity evolution during Portuguese red wine vinification and their relation with the proanthocyanidin and anthocyanin composition. J. Food Process. Preserv. 2012, 36, 298-309. [CrossRef]

2. Pajović, R.; Raičević, D.; Popović, T.; Sivilotti, P.; Lisjak, K.; Vanzo, A. Polyphenolic characterisation of Vranac, Kratosija and Cabernet Sauvignon (Vitis vinifera L. cv.) grapes and wines from different vineyard locations in Montenegro. S. Afr. J. Enol. Vitic. 2014, 35, 134-143. [CrossRef]

3. Ivanova-Petropulos, V.; Hermosín-Gutiérrez, I.; Boros, B.; Stefova, M.; Stafilov, T.; Vojnoski, B.; Kilár, F. Phenolic compounds and antioxidant activity of Macedonian red wines. J. Food Compos. Anal. 2015, 41, 1-14. [CrossRef]

4. De Pascali, S.A.; Coletta, A.; Del Coco, L.; Basile, T.; Gambacorta, G.; Fanizzi, F.P. Viticultural practice and winemaking effects on metabolic profile of Negroamaro. Food Chem. 2014, 161, 112-119. [CrossRef] [PubMed]

5. Raičević, D.; Božinović, Z.; Petkov, M.; Ivanova-Petropulos, V.; Kodžulović, V.; Mugoša, M.; Šućur, S.; Maraš, V. Polyphenolic content and sensory profile of Montenegrin Vranac wines produced with different oenological products and maceration. Maced. J. Chem. Chem. Eng. 2017, 36, 229-238. [CrossRef]

6. Rusjan, D.; Veberič, R.; Mikulič-Petkovšek, M. The response of phenolic compounds in grapes of the variety 'Chardonnay' (Vitis vinifera L.) to the infection by phytoplasma Bois noir. Eur. J. Plant Pathol. 2012, 133, 965-974. [CrossRef]

7. Bešlić, Z.; Pantelić, M.; Dabić, D.; Todić, S.; Natić, M.; Tešić, Ž. Effect of vineyard floor management on water regime, growth response, yield and fruit quality in Cabernet Sauvignon. Sci. Hortic. (Amsterdam, Neth.) 2015, 197, 650-656. [CrossRef]

8. Bindon, K.; Varela, C.; Kennedy, J.; Holt, H.; Herderich, M. Relationships between harvest time and wine composition in Vitis vinifera L. cv. Cabernet Sauvignon 1. Grape and wine chemistry. Food Chem. 2013, 138, 1696-1705. [CrossRef]

9. Maletić, E.; Kontić, J.K.; Preiner, D.; Jeromel, A.; Patz, C.-D.; Dietrich, H. Anthocyanin profile and antioxidative capacity of some autochthonous Croatian red wines. J. Food, Agric. Environ. 2009, 7, 48-51.

10. Laurentiu, M.P.; Popa, M.E. Polyphenol fingerprinting approaches in wine traceability and authenticity: Assessment and implications of red wines. Beverages 2018, 4, 75-92.

11. Dimitrovska, M.; Bocevska, M.; Dimitrovski, D.; Doneva-Sapceska, N. Evolution of anthocyanins during vinification of merlot and pinot noir grapes to wines. Acta Aliment. 2015, 44, 259-267. [CrossRef]

12. Zhao, Q.; Duan, C.Q.; Wang, J. Anthocyanins profile of grape berries of Vitis amurensis, its hybrids and their wines. Int. J. Mol. Sci. 2010, 11, 2212-2228. [CrossRef] [PubMed]

13. Pantelić, M.; Dabić Zagorac, D.; Davidović, S.; Todić, S.; Bešlić, Z.; Gašić, U.; Tešić, Ž.; Natić, M. Identification and quantification of phenolic compounds in berry skin, pulp, and seeds in 13 grapevine varieties grown in Serbia. Food Chem. 2016, 211, 243-252. [CrossRef] [PubMed]

14. Pantelić, M.; Dabić Zagorac, D.; Ćirić, I.; Pergal, M.; Relić, D.; Todić, S.; Natić, M. Phenolic profiles, antioxidant activity and minerals in leaves of different grapevine varieties grown in Serbia. J. Food Compos. Anal. 2017, 62, 76-83.

15. Pantelić, M.; Dabić Zagorac, D.; Natić, M.; Gašić, U.; Jović, S.; Vujović, D.; Popović Djordjević, J. Impact of clonal variability on phenolics and radical scavenging activity of grapes and wines: A study on the recently developed Merlot and Cabernet Franc clones (Vitis vinifera L.). PLoS ONE 2016, 11, e0163823. [CrossRef]

16. Pešić, M.; Milinčić, D.; Kostić, A.; Stanisavljević, N.; Vukotić, G.; Kojić, M.; Gašić, U.; Barać, M.; Stanojević, S.; Popović, D.; et al. In vitro digestion of of meat- and cereal-based food matrix enriched with grape extracts: How are polyphenol composition, bioaccessibility and antioxidant activity affected? Food Chem. 2019, 284, 28-44. [CrossRef]

17. Đorđević, N.; Novaković, M.; Pejin, B.; Živković, M.; Savić, A.; Mutić, J.; Tešević, V. An insight into chemical composition and biological activity of Montenegrin Vranac red wine. Sci. Hortic. (Amst. Neth.) 2018, 230, 142-148. [CrossRef]

18. Radovanović, B.; Radovanovic, A.; Tomić, V. Relations between the phenolic composition and free radical scavenging, and antibacterial activities of red wines from different cultivars of Vitis vinifera L. Int. J. Food Prop. 2012, 15, 725-735. [CrossRef] 
19. Matić, P.; Sabljić, M.; Jakobek, L. Validation of spectrophotometric methods for the determination of total polyphenol and total flavonoid content. J. AOAC Int. 2017, 100, 1795-1803. [CrossRef]

20. Radovanović, A.; Radovanović, B.; Jovančićević, B. Free radical scavenging and antibacterial activities of southern Serbian red wines. Food Chem. 2009, 117, 326-331. [CrossRef]

21. Mitić, M.; Kostić, D.; Pavlović, A.; Micić, R.; Stojanović, B.; Paunović, D.; Dimitrijević, D. Antioxidant activity and polyphenol profile of Vranac red wines from Balkan region. Hem. Ind. 2016, 70, 265-275. [CrossRef]

22. Pantelić, M.; Dabić, D.; Matijašević, S.; Davidović, S.; Dojčinović, B.; Milojković-Opsenica, D.; Tešić, Ž.; Natić, M. Chemical characterization of fruit wine made from oblačinska sour cherry. Sci. World J. 2014, 2014, 454797. [CrossRef] [PubMed]

23. Oliveira, J.; Da Silva, M.A.; Teixeira, N.; De Freitas, V.; Salas, E. Screening of anthocyanins and anthocyanin-derived pigments in red wine grape pomace using LC DAD/MS and MALDI-TOF techniques. J. Agric. Food Chem. 2015, 61, 7616-7644. [CrossRef] [PubMed]

24. Barnaba, C.; Dellacassa, E.; Nicolini, G.; Giacomelli, M.; Villegas, T.R.; Nardin, T.; Larcher, R. Targeted and untargeted high resolution mass approach for a putative profiling of glycosylated simple phenols in hybrid grapes. Food Res. Int. 2017, 98, 20-33. [CrossRef] [PubMed]

25. Teixeira, A.; Eiras-Dias, J.; Castellarin, S.D.; Gerós, H. Berry phenolics of grape-vine under challenging environments. Int. J. Mol. Sci. 2013, 14, 18711-18739. [CrossRef] [PubMed]

26. Liang, N.; Kitts, D.D. Role of chlorogenic acids in controlling oxidative and inflammatory stress conditions. Nutrients 2016, 8, 16. [CrossRef]

27. Yuzuak, S.; Ballington, J.; Xie, D.Y. HPLC-qTOF-MS/MS-based profiling of flavan-3-ols and dimeric proanthocyanidins in berries of two muscadine grape hybrids FLH 13-11 and FLH 17-66. Metabolites 2018, 8, 57. [CrossRef]

28. Chen, J.; Xu, Z.; Zhu, W.; Nie, R.; Li, C. Novel proanthocyanidin dimer analogues with the C-ring-opened diaryl-propan-2-gallate structural unit and enhanced antioxidant activities. J. Funct. Foods 2016, 21, 290-300. [CrossRef]

29. Ridder, L.; van der Hooft, J.; Verhoevem, S.; de Vos, R.C.H.; Bino, R.J.; Vervoort, J. Automatic chemical structure annotation of an LC-MS based metabolic profilefrom green tea. Anal. Chem. 2013, 85, 6033-6040. [CrossRef]

30. Ma, W.; Waffo-Téguo, P.; Jourdes, M.; Li, H.; Teissedre, P.L. First evidence of epicatechin vanillate in grape seed and red wine. Food Chem. 2018, 259, 304-310. [CrossRef]

31. Mattivi, F.; Guzzon, R.; Vrhovšek, U.; Stefanini, M.; Velasco, R. Metabolite profiling of grape: Flavanols and anthocyanins. J. Agric. Food Chem. 2006, 54, 7692-7702. [CrossRef] [PubMed]

32. Schoedl, K.; Schuhmacher, R.; Forneck, A. Studying the polyphenols of grapevine leaves according to age and insertion level under controlled conditions. Sci. Hortic. (Amst. Neth.) 2012, 141, 37-41. [CrossRef]

33. Fernandes, F.; Ramalhosa, E.; Pires, P.; Verdial, J.; Valentão, P.; Andrade, P.; Bento, A.; Albert Pereira, J. Vitis vinifera leaves towards bioactivity. Ind. Crops Prod. 2013, 43, 434-440. [CrossRef]

34. Chen, Q.; Diao, L.; Song, H.; Zhu, X. Vitis amurensis Rupr: A review of chemistry and pharmacology. Phytomedicine 2018, 49, 111-122. [CrossRef] [PubMed]

35. He, F.; Liang, N.N.; Mu, L.; Pan, Q.H.; Wang, J.; Reeves, M.J.; Duan, C.Q. Anthocyanins and their variation in red wines I. Monomeric anthocyanins and their color expression. Molecules 2012, 17, 1571-1601. [CrossRef] [PubMed]

36. Kim, M.; Yoon, S.H.; Jung, M.; Choe, E. Stability of meoru (Vitis coignetiea) anthocyanins under photochemically produced singlet oxygen by riboflavin. New Biotechnol. 2010, 27, 435-439. [CrossRef] [PubMed]

37. He, F.; Liang, N.N.; Mu, L.; Pan, Q.H.; Wang, J.; Reeves, M.J.; Duan, C.Q. Anthocyanins and their variation in red wines. II. Anthocyanin derived pigments and their color evolution. Molecules 2012, 17, 1483-1519. [CrossRef]

38. De Freitas, V.; Mateus, N. Formation of pyranoanthocyanins in red wines: A new and diverse class of anthocyanin derivatives. Anal. Bioanal. Chem. 2011, 401, 1463-1473. [CrossRef]

39. Mitić, M.N.; Kostić, D.A.; Pavlović, A.N. The phenolic composition and the antioxidant capacity of Serbian red wines. Adv. Techn. 2014, 3, 16-22. 